\title{
Higher Blood Pressure during Endovascular Thrombectomy in Anterior Circulation Stroke Is Associated with Better Outcomes
}

\author{
Slaven Pikija, ${ }^{a}$ Vladimir Trkulja, ${ }^{\mathrm{b}}$ Christian Ramesmayer, ${ }^{\mathrm{a}}$ Johannes S. Mutzenbach, ${ }^{\mathrm{a}}$ \\ Monika Killer-Oberpfalzer, ${ }^{\mathrm{a}, \mathrm{c}}$ Constantin Hecker, ${ }^{\mathrm{a}}$ Nele Bubel, ${ }^{\mathrm{a}}$ Michael Ulrich Füssel, ${ }^{\mathrm{d}}$ Johann Sellner ${ }^{\mathrm{a}, \mathrm{e}}$ \\ ${ }^{a}$ Department of Neurology, Christian Doppler Medical Center, Paracelsus Medical University, Salzburg, Austria \\ ${ }^{b}$ Department for Pharmacology, School of Medicine, University of Zagreb, Zagreb, Croatia \\ Institute for Neurointervention, Paracelsus Medical University, Salzburg, Austria \\ dInstitute of Neuroanesthesiology, Christian Doppler Medical Center, Paracelsus Medical University, Salzburg, Austria \\ 'Department of Neurology, Rechts der Isar Hospital, Technical University of Munich, Munchen, Germany
}

Background and Purpose Reports investigating the relationship between in-procedure blood pressure (BP) and outcomes in patients undergoing endovascular thrombectomy (EVT) due to anterior circulation stroke are sparse and contradictory.

Methods Consecutive EVT-treated adults (modern stent retrievers, BP managed in line with the recommendations, general anesthesia, invasive BP measurements) were evaluated for associations of the rate of in-procedure systolic BP (SBP) and mean arterial pressure (MAP) excursions to $>120 \% /<80 \%$ of the reference values (serial measurements at anesthesia induction) and of the reference $\mathrm{BP} /$ weighted in-procedure mean BP with post-procedure imaging outcomes (ischemic lesion volume [ILV], hemorrhages) and 3-month functional outcome (modified Rankin Scale [mRS], score 0 to 2 vs. 3 to 6$)$.

Results Overall 164 patients (70.7\% pharmacological reperfusion, 80.5\% with good collaterals, 73.8\% with successful reperfusion) were evaluated for ILV (range, 0 to $581 \mathrm{~cm}^{3}$ ) and hemorrhages (incidence 17.7\%). Higher rate of in-procedure SBP/MAP excursions to $>120 \%$ was independently associated with lower ILV, while higher in-procedure mean SBP/MAP was associated with lower odds of hemorrhages. mRS 0-2 was achieved in 75/155 (48.4\%) evaluated patients (nine had missing mRS data). Higher rate of SBP/MAP excursions to $>120 \%$ and higher reference SBP/MAP were independently associated with higher odds of $\mathrm{mRS} 0-2$, while higher ILV was associated with lower odds of mRS 0-2. Rate of SBP/MAP excursions to $<80 \%$ was not associated with any outcome.

Conclusions In the EVT-treated patients with BP managed within the recommended limits, a better functional outcome might be achieved by targeting in-procedure BP that exceeds the preprocedure values by more than $20 \%$.
Correspondence: Slaven Pikija Department of Neurology, Christian Doppler Medical Center, Paracelsus Medical University, Ignaz-Harrer-Straße 79, 5020 Salzburg, Austria Tel: +43-676-403-5616 Fax: +43-5-7255-30399 E-mail:s.pikija@salk.at

Received: May 3, 2018

Revised: September 17, 2018

Accepted: September 17, 2018

Keywords Stroke; Mechanical thrombolysis; Blood pressure; Anesthesia, general 


\section{Introduction}

Critical blood pressure (BP) values in the acute ischemic stroke patients undergoing reperfusion treatment are based on the exclusion criteria in the pivotal Phase III trials of recombinant human tissue plasminogen activator (rtPA). ${ }^{1,2}$ These BP limits were applied in the most recent endovascular thrombectomy (EVT) studies. ${ }^{3}$ However, there have been no randomized controlled trials specifically evaluating the proposed BP limits and there is uncertainty whether BP level of $185 / 105 \mathrm{~mm} \mathrm{Hg}$ as an exclusionary criterion for reperfusion treatments can be generalized. ${ }^{4}$ Notably, studies in hypertensive patients demonstrated a shift of the limits of functional cerebral blood flow autoregulation to higher values in patients with higher pre-stroke BP levels. ${ }^{5,6}$ Thus, keeping BP at a certain universally defined level during the most vulnerable phase of acute cerebral ischemia seems counterintuitive. In animals models of stroke, cerebral blood flow in moderately under-perfused tissue depends on systemic BP and any significant drop in BP is likely to compromise penumbra viability. ${ }^{7}$ However, BP beyond the proposed limits could be detrimental due to higher risk of post-reperfusion hemorrhages after EVT. ${ }^{8}$ Oscillations in systolic blood pressure (SBP) after EVT may also be associated with poorer outcomes. ${ }^{9}$ Earlier studies suggested U-shaped associations between on-admission BP and favorable clinical outcomes in ischemic stroke patients, but also poorer outcomes in patients with generally higher BP throughout hospitalizations. ${ }^{10-12}$ One recent analysis reported an association between higher maximum in-procedure SBP and poor 30-day outcomes, while several others suggested the opposite-associations between poorer outcomes and indicators of BP dips. ${ }^{13-16}$ Thus, we hypothesized that during EVT it would be reasonable to target individualized BP values. We therefore investigated the relationship between in-procedure SBP and mean arterial pressure (MAP) excursions to above or below the limits defined in respect to their pre-procedure values and (1) post-procedure imaging findings: ischemic lesion volume (ILV) and visible hemorrhages; (2) 3-month functional outcomes; in a cohort of adults with anterior circulation ischemic stroke treated with EVT.

\section{Methods}

\section{Design}

This is a retrospective analysis of a single-center prospective database comprising adults (age $\geq 18$ years) with a symptomatic acute ischemic stroke due to occlusion of the internal carotid artery and/or middle cerebral artery treated with EVT using modern stent retrievers (a few patients received thrombus aspiration only) over a 5-year period (January 1, 2012 to December 31,2016$)$. It was approved by the Ethics Committee of Bundesland Salzburg. Figure 1A depicts the flow: patients underwent EVT under general anesthesia (GA) with or without intravenous rtPA, which individually required measures for $\mathrm{BP}$ reduction; ${ }^{4}$ the analysis was restricted to patients with invasive BP measurement; serial BP values taken immediately before the start of EVT/induction of anesthesia were considered a "reference BP" for the period of the procedure; patients underwent post-procedure computed tomography (CT) imaging to assess hemorrhages and ILV, and were evaluated for the functional outcome at 3 months post-stroke (modified Rankin Scale

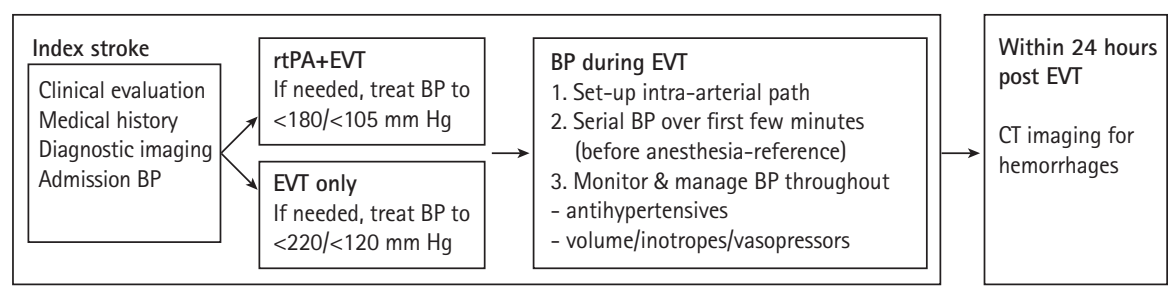

\begin{tabular}{|c|c|c|c|}
\hline \multicolumn{2}{|c|}{ Covariates } & \multicolumn{1}{|c|}{ Primary clinical endpoint } \\
\cline { 1 - 2 } $\begin{array}{l}\text { In-procedure BP and relative measures of } \\
\text { oscillations vs. the reference values }\end{array}$ & Predict? & $\begin{array}{l}\text { Post-procedure CT findings: visible hemorrhages, } \\
\text { ischemic lesion volume }\end{array}$ & $\begin{array}{l}\text { Functional outcome at 3 months: } \\
\text { favorable (modified Rankin Scale score 0-2) }\end{array}$ \\
\hline "Baseline" predictors & Intermediate outcomes/predictors-mediators? & Relationship? \\
\hline
\end{tabular}

Figure 1. (A) Patient flow. (B) Steps in data analysis. We analyzed relationships between blood pressure (BP) during endovascular thrombectomy (EVT) with or without recombinant tissue plasminogen activator (rtPA) with (1) post-procedure computed tomography (CT) findings: ischemic lesion volume (ILV) and visible hemorrhages; (2) functional outcome at 3 months. We explored a possibility of a mediated association: in-procedure BP $\rightarrow$ ILV/visible hemorrhage $\rightarrow$ 3-month functional outcome. 
[mRS]). We explored the associations between in-procedure BP oscillations defined in relative terms versus the "reference value" and (1) post-procedure ILV and visible hemorrhages; (2) favorable 3-month functional outcome (mRS 0-2) (Figure 1B).

\section{Clinical evaluations}

Stroke severity was assessed using the National Institutes of Health Stroke Scale (NIHSS). Indication for EVT was made in line with continuously updated standards, while indication for rtPA was in accordance with the Safe Implementation of Thrombolysis in Stroke-Monitoring Study. ${ }^{2,17}$ At discharge, stroke etiology was classified according to the Trial of Org 10172 in Acute Stroke Treatment (TOAST) criteria. ${ }^{18}$ Functional outcome at 3 months was assessed at scheduled visits or by a telephone interview with the patient or a proxy.

\section{Radiological evaluations}

CT or magnetic resonance was used to identify the vessel(s) portions occluded and to evaluate leptomeningeal collaterals on CT-angiography. Collaterals were graded as good when $\geq 50 \%$ were present as compared to the unaffected side. Postprocedure CT was used to evaluate presence of hemorrhages and last available CT during the index hospitalization to determine ILV. ${ }^{19}$ The reperfusion success was graded by the Thrombolysis in Cerebral Infarction (TICl) scale: grades $2 b$ (perfusion $>50 \%$ of the vascular distribution of the occluded artery) and 3 (full perfusion with filling of all distal branches) were considered a success, grades $0-2 a$ were considered a failure. ${ }^{20}$

\section{BP measurements and management}

During EVT, BP was measured invasively every minute (except in two patients: every 2 or 5 minutes) and recordings were saved by the MetaVision ${ }^{\circledR}$ software (iMDsoft, Düsseldorf, Germany). Otherwise, it was measured regularly (cuff technique) and recorded in the patient charts. The in-house algorithm for acute stroke patients has been constantly in agreement with the respective guidelines, particularly in respect to the use of rtPA and EVT.

\section{BP indicators}

We determined the following BP indicators: (1) "reference" SBP/MAP as the mean of 3 to 5 measurements taken immediately before the start of EVT (induction of anesthesia); (2) individual values $\pm 20 \%$ of the reference were determined and number of in-procedure excursions to values $>120 \%$ or to $<80 \%$ of the reference was counted to calculate their rates (episodes/10 minutes); (3) weighted mean in-procedure SBP/ MAP by dividing area under the curve of BP over time (first to last in-procedure measurement) by the time period covered. We also determined rates of any excursions exceeding $\pm 20 \%$ of the reference, and absolute (by linear interpolation) and relative (percent) time of the procedure with SBP/MAP $>120 \%$ or $<80 \%$ of the reference values.

\section{Data analysis}

We first evaluated associations of in-procedure BP with postprocedure ILV and hemorrhages, and then with 3-month mRS 0-2 (Figure 1B). General linear models were fitted to In-transformed ILV (effects are geometric mean ratio [GMR], exponents of coefficient obtained with logarithms) and logistic models were fitted to hemorrhages and mRS $0-2$. We considered rates of excursions to $>120 \%$ and to $<80 \%$ of the reference (analyzed jointly due to potential off-setting effects) to be the primary indicators of BP oscillations, as they were determined directly. Analyses based on derived indicators (absolute/relative time) provided virtually identical results, and are not shown. Analyses of the rate of any excursions exceeding $\pm 20 \%$ provided no additional information (also not shown).

Several models were fitted to each outcome starting with larger ("full") models (default and selected effects [stepwise selection with $P<0.200$ to enter/stay] with sequential tests of interactions: excursion rates*reference $\mathrm{BP}$, excursion rates* $\mathrm{TICl}$ grade, reference $\mathrm{BP} / \mathrm{in}$-procedure mean $\mathrm{BP} * \mathrm{TICl}$ grade), from which "reduced" models (to avoid overfitting) were derived (effects of primary interest and biologically/statistically plausible adjustments). In the analysis of 3-month mRS, intermediate outcomes (ILV, hemorrhages) were introduced as adjustments into the "reduced" models, so model selection included a further step to "final" models. Figure 2 outlines the model selection procedure. For details on multivariate model building and selection see Supplementary Methods. Mediation analysis ${ }^{21}$ evaluated possibility that the effects of BP on the functional outcome were mediated "through" the effects on ILV/hemorrhages. We used SAS version 9.4 statistical software (SAS Inc., Cary, NC, USA).

\section{Results}

\section{Patients}

Of the 202 admitted patients, 20 did not undergo EVT and 18 did not have invasive BP monitoring. The cohort analyzed for ILV and hemorrhages comprised 164 patients, while 155 were analyzed for 3-month mRS (nine patients lacked data) (Figure 3).

Patient characteristics are summarized in Table 1 (Supplementary Table 1 for laboratory tests). Both on-admission and reference SBP/MAP varied greatly. Reference values appeared 


\begin{tabular}{|c|c|c|c|}
\hline \multicolumn{3}{|c|}{ Intermediate (computed tomography [CT]) outcomes } & \multirow{2}{*}{$\begin{array}{l}\text { Primary clinical endpoint } \\
\text { mRS at } 3 \text { months 0-2 (vs. 3-6) }\end{array}$} \\
\hline Types of models & Ischemic lesion volume (ILV) & Post-procedure visible hemorrhages & \\
\hline $\begin{array}{l}\text { FULL } \\
\text { Initial: default and selected independents, } \\
\text { main effects, no interactions } \\
\text { Sequentially testing interactions: } \\
\text { a) rate of BP excursions*reference BP; } \\
\text { b) rate of excursions*IICl grade; } \\
\text { c) reference } B P * * I C l, \text { in-procedure mean } \\
\text { BP*IICI }\end{array}$ & $\begin{array}{l}\text { - Default: rate of BP excursions to }>120 \% \\
\text { of the reference, rate of excursions to }<80 \% \text {, } \\
\text { reference BP, in-procedure mean BP, EVT } \\
\text { duration, number of BP measurements taken, } \\
\text { on-admission NIHSS score, TICI grade, } \\
\text { affected vessel, collaterals } \geq 50 \% \text { as } \\
\text { unaffected side, use of antihypertensives } \\
\text { before EVT } \\
\text { - Selected: sex }\end{array}$ & $\begin{array}{l}\text { - Default: rate of BP excursions to }>120 \% \\
\text { of the reference, rate of excursions to }<80 \% \text {, } \\
\text { reference } \mathrm{BP}, \text { in-procedure mean } \mathrm{BP}, \mathrm{EV} \\
\text { duration, number of } \mathrm{BP} \text { measurements taken, } \\
\text { on-admission NIHSS score, TICl grade, use of } \\
\text { antihypertensives before EVT } \\
\text { - Selected: stroke etiology, history of } \\
\text { coronary artery disease }\end{array}$ & $\begin{array}{l}\text { - Default: rate of BP excursions to }>120 \% \\
\text { of the reference, rate of excursions to }<80 \% \text {, } \\
\text { reference BP, in-procedure mean BP, EVT } \\
\text { duration, number of BP measurements taken, } \\
\text { on-admission NIHSS score, TICl grade, } \\
\text { collaterals } \geq 50 \% \text { as unaffected side, use of } \\
\text { antihypertensives before EVT } \\
\text { - Selected: stroke etiology, pre-/post-stroke } \\
\text { statin use, age }\end{array}$ \\
\hline $\begin{array}{l}\text { REDUCED } \\
\text { Keep: a) excursion rates (primary interest); } \\
\text { b) biologically \& statistically plausible } \\
\text { other effects (default or selected, } \\
\text { interactions) }\end{array}$ & $\begin{array}{l}\text { Kept effects: rate of BP excursions to } \\
>120 \% \text {, rate of BP excursions to }<80 \% \text {, } \\
\text { reference BP, rate to }>120 \% * * \text { reference BP } \\
\text { interaction, NIHSS score, TICl grade, } \\
\text { collaterals, sex }\end{array}$ & $\begin{array}{l}\text { Kept effects: rate of BP excursions to } \\
>120 \% \text {, rate of BP excursions to }<80 \% \text {, } \\
\text { reference BP, in-procedure mean BP, NIHSS } \\
\text { score, TICI grade, stroke etiology, history of } \\
\text { coronary artery disease }\end{array}$ & $\begin{array}{l}\text { A. Without interaction and CT outcomes } \\
\text { Kept effects: rate of BP excursions to }>120 \% \\
\text { rate of BP excursions to }<80 \% \text {, reference } \mathrm{BP} \text {, } \\
\text { in-procedure mean BP, NIHSS score, TICl } \\
\text { grade, collaterals, stroke etiology, }\end{array}$ \\
\hline \multirow{6}{*}{$\begin{array}{l}\text { FINAL } \\
\text { Further model reduction by final exclusion } \\
\text { of the initial default effects and } \\
\text { apparently irrelevant CT findings }\end{array}$} & & & $\begin{array}{l}\text { B. Without interaction, with CT outcomes } \\
\text { As A+ILV and hemorrhages }\end{array}$ \\
\hline & & & $\begin{array}{l}\text { C. With interaction, without CT outcomes } \\
\text { As A+rate to }>120 \% * \text { ICl interaction } \\
\text { D. With interaction and CT outcomes } \\
\text { As C+ILV and hemorrhages }\end{array}$ \\
\hline & & & $\begin{array}{l}\text { A. Without interaction and CT outcomes } \\
\text { Kept effects: rate of BP excursions to }>120 \% \\
\text { rate of BP excursions to }<80 \% \text {, reference BP, } \\
\text { NIHSS score, TICI grade, collaterals, stroke } \\
\text { etiology, pre-/post-stroke statin use, age }\end{array}$ \\
\hline & & & $\begin{array}{l}\text { B. Without interaction and CT outcomes } \\
\text { As A+ILV }\end{array}$ \\
\hline & & & $\begin{array}{l}\text { C. With interaction, Without CT outcomes } \\
\text { As A+rate to }>120 \% *{ }^{\prime} I C \text { interaction }\end{array}$ \\
\hline & & & $\begin{array}{l}\text { D. With interaction and CT outcomes } \\
\text { As C+ILV }\end{array}$ \\
\hline
\end{tabular}

Figure 2. Model selection strategy. All models were fitted separately for systolic blood pressure (BP) and mean arterial pressure. TICl, Thrombolysis in Cerebral Infarction; EVT, endovascular thrombectomy; NIHSS, National Institutes of Health Stroke Scale; mRS, modified Rankin Scale.

considerably lower than on-admission values, although intraindividual differences extended from substantial reduction to a substantial increase (Table 1). A total of 16 patients received antihypertensives between admission and EVT and two received sympathomimetics. Procedure lasted 12 to 587 minutes with 13 to $230 \mathrm{BP}$ measurements, and 77 (46.9\%) patients received sympathomimetics during EVT (Table 1). Overall, 38/164 (23.2\%) patients had no excursions exceeding $\pm 20 \%$ of the reference SBP/MAP, 68 and 70 (41.5\% and $43.6 \%$ for SBP and MAP, respectively) experienced exclusively excursions to $>120 \%, 37$ and 31 (22.6\% and 18.9\%) exclusively excursions to $<80 \%$, and 21 and 25 ( $12.8 \%$ and $15.2 \%)$ experienced both. Data are summarized in Table 1 (see also Supplementary Table 2 and Supplementary Figure 1; for absolute/relative time $>120 \%$ and $<80 \%$ of the reference). Additional BP analysis (Supplementary Analysis of Blood Pressure) indicated that BPrelated procedures were guided by the intention to keep $B P$ within the recommended limits, and not by the presenting stroke characteristics.

\section{Relationship between in-procedure BP and ILV/ post-procedure hemorrhages}

Of the 164 patients, $132(80.5 \%)$ had good collaterals, 121 (73.8\%) achieved successful reperfusion, ILV ranged 0 to $518 \mathrm{~cm}^{3}$ and $29(17.7 \%)$ patients had visible hemorrhages (Table 1). Full models were fitted to each outcome (see Supplementary Tables 3 and 4 for complete models). Independent variables consistently not associated with the outcomes were consecutively removed (Figure 2). Reduced models are shown in Table 2. Higher rate of in-procedure SBP excursions to $>120 \%$ of the reference was associated with lower ILV, more so at higher reference values (rate*reference interaction) (Table 2). As an illustration, with reference $\mathrm{SBP}<120 \mathrm{~mm} \mathrm{Hg}(\mathrm{n}=62)$ the adjusted $\mathrm{GMR}=0.89$ (interquartile range [IQR], 0.74 to 1.08), with reference $\mathrm{SBP}>135 \mathrm{~mm}$ $\mathrm{Hg}(\mathrm{n}=60), \mathrm{GMR}=0.34$ (IOR, 0.15 to 0.74$)$. The effect of SBP excursions was consistent in patients with $\mathrm{TICl} 0-2 \mathrm{a}(\mathrm{n}=43)$ and $\mathrm{TICl} 2 \mathrm{~b}-3$ ( $n=121$ ) (Supplementary Table 3). SBP excursions to $<80 \%$ of the reference were not associated with ILV. Higher admission NIHSS score and male sex were associated with higher $\mathrm{ILV} ; \mathrm{TICl}$ grade $2 \mathrm{~b}-3$ and good collaterals were associated with 


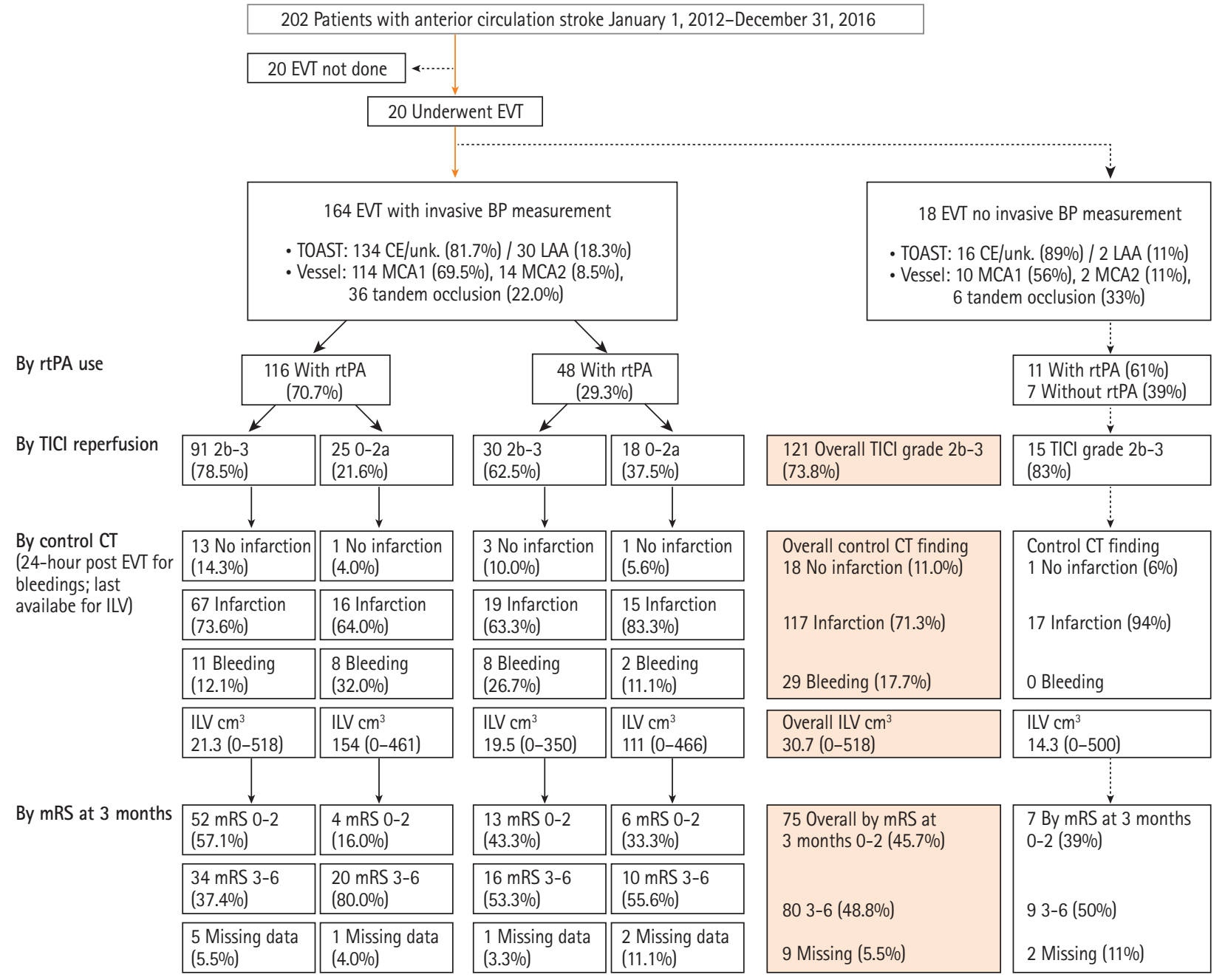

Figure 3. Disposition of patients. EVT, endovascular thrombectomy; BP, blood pressure; TOAST, Trial of Org 10172 in Acute Stroke Treatment classification; CE, cardioembolic; unk., unknown etiology; LAA, large artery atherosclerosis; MCA, middle cerebral artery, segment 1, segment 2; rtPA, recombinant human tissue plasminogen activator; $\mathrm{TICl}$, Thrombolysis in Cerebral Infarction; CT, computed tomography; ILV, ischemic lesion volume; mRS, modified Rankin Scale.

lower ILV. Similar associations were observed for MAP, except for the excursions*reference interaction (Table 2).

In-procedure BP oscillations assessed by BP excursions were not associated with the post-procedure hemorrhages, higher reference SBP/MAP tended towards higher odds, while higher in-procedure mean SBP/MAP was associated with lower odds of hemorrhages (Table 2 and Supplementary Table 4). Higher in-procedure SBP/MAP was determined mainly by the higher reference SBP/MAP (Supplementary Analysis of Blood Pressure, Table F). Further analysis demonstrated that higher reference SBP/MAP was indirectly (via in-procedure mean) associated with lower odds of post-procedure hemorrhages: the indirect effect was practically identical in size to the direct effect (Table 2); hence, the total effect was close to zero (Supplementary Analysis of Post-Procedure Hemorrhages).

\section{Relationship between in-procedure BP and} 3-month functional outcome

Patients with mRS 0-2 (75/155, 48.4\%) appeared younger, were more commonly men, and less commonly suffered comorbidities than the patients with mRS 3-6 (Table 1). They were more frequently pre-index stroke statin users, and less frequently antiplatelets or anticoagulants users (Table 1). On-admission NIHSS score, prevalence of cardioemobolic/unknown etiology and of tandem occlusions were lower while the use of rtPA was more common and the onset-to-EVT time was shorter in patients with mRS 0-2 (Table 1). Average on-admission and reference SBP/MAP appeared similar between the two subsets and similarly low numbers of patients received antihypertensives between admission and EVT. BP decline between admission and EVT appeared somewhat greater in patients with mRS 3-6 (Table 1). Weighted mean in-proce- 
Table 1. Patient and procedure characteristics, overall and by 3-month functional outcome*

\begin{tabular}{|c|c|c|c|c|}
\hline Characteristic & All patients & $\mathrm{mRS} 0-2$ & $m R S 3-6$ & Missing mRS \\
\hline Number & 164 & 75 & 80 & 9 \\
\hline Age (yr) & $74(20-92)$ & $67(20-91)$ & 79 (44-92) & $74(52-89)$ \\
\hline Male sex & $73(44.5)$ & $37(49.3)$ & $31(38.8)$ & $5(55.6)$ \\
\hline Atrial fibrillation & $64(39.0)$ & $23(30.7)$ & $36(45.0)$ & $5(55.6)$ \\
\hline Previous stroke & $18(11.0)$ & $8(11.0)$ & $8(10.0)$ & $2(22.2)$ \\
\hline Peripheral artery disease & $11(6.7)$ & $4(5.3)$ & $7(8.8)$ & $0(0)$ \\
\hline Ischemic heart disease & $35(21.3)$ & $9(12.0)$ & $25(31.3)$ & $1(11.1)$ \\
\hline Carotid stenosis $\geq 50 \%$ & $17(10.4)$ & $8(10.7)$ & $8(10.0)$ & $1(11.1)$ \\
\hline Hypertension & $106(64.6)$ & $41(54.7)$ & 59 (73.8) & $6(66.7)$ \\
\hline Diabetes mellitus & $21(12.8)$ & $7(9.3)$ & $13(16.3)$ & $1(11.1)$ \\
\hline Chronic heart failure & $26(16.2)$ & $8(11.0)$ & $18(22.8)$ & $0(0)$ \\
\hline Chronic renal failure & $16(9.9)$ & $4(56.42)$ & $12(15.2)$ & $0(0)$ \\
\hline Pre-admission statins & $135(82.8)$ & $68(90.7)$ & $60(75.0)$ & $1(87.5)$ \\
\hline Pre-admission antiplatelets & $45(27.4)$ & $15(20.0)$ & $28(35.0)$ & $2(22.2)$ \\
\hline Pre-admission anticoagulants & $29(17.7)$ & $9(12.0)$ & $18(22.5)$ & $2(22.2)$ \\
\hline On-admission systolic BP (mm Hg) & 150 (83-223) & $150(100-210)$ & 154 (83-223) & $148(110-192)$ \\
\hline On-admission MAP (mm Hg) & $107(56-174)$ & 106 (67-143) & $108(56-174)$ & 106 (70-135) \\
\hline NIHSS at presentation & $18(3-32)$ & $16(3-31)$ & $20(3-32)$ & $18(7-24)$ \\
\hline Onset to first vessel imaging (min) & 189 (98-900) & $181(98-374)$ & 208 (99-639) & $165(111-900)$ \\
\hline TOAST: cardioembolic or unknown & $134(81.7)$ & $54(72.0)$ & $72(90.0)$ & $8(88.9)$ \\
\hline rtPA used & 116 (70.7) & $56(74.7)$ & $54(67.5)$ & $6(66.7)$ \\
\hline Middle cerebral artery segment 1 & $114(69.5)$ & $52(69.3)$ & $55(68.8)$ & $7(77.8)$ \\
\hline Middle cerebral artery segment 2 & $14(8.5)$ & $8(10.7)$ & $4(5.0)$ & $2(22.2)$ \\
\hline Tandem occlusion & $36(22.0)$ & $15(20.0)$ & $21(26.3)$ & $0(0)$ \\
\hline Procedure duration (min) & $83(12-587)$ & $65(12-202)$ & 109 (17-587) & $72(17-188)$ \\
\hline BP measurements/procedure (n) & $83(13-230)$ & $66(13-203)$ & 108 (18-230) & $73(18-189)$ \\
\hline $\mathrm{BP}$ measurements/minute & $1.0(0.2-2.4)$ & $1.0(0.5-2.4)$ & $1.0(0.2-1.6)$ & $1.0(1.0-1.0)$ \\
\hline SBP reference $(\mathrm{mm} \mathrm{Hg})$ & 125 (73-203) & 125 (93-203) & $124(73-191)$ & 132 (98-182) \\
\hline $\mathrm{SBP} \Delta$ reference-admission $(\mathrm{mm} \mathrm{Hg})$ & $-23(-126$ to 66$)$ & -19 (-88 to 43$)$ & $-28(-126$ to 66$)$ & $-23(-53$ to 37$)$ \\
\hline MAP reference (mm Hg) & $88(56-136)$ & $91(61-136)$ & $87(56-134)$ & $84(64-123)$ \\
\hline MAP $\Delta$ reference-admission $(\mathrm{mm} \mathrm{Hg})$ & -17 (-101 to 50$)$ & $-15(-56$ to 46$)$ & $-18(-101$ to 50$)$ & $-22(-55$ to 21$)$ \\
\hline \multicolumn{5}{|l|}{$\mathrm{BP}$ treatment admission-to-procedure } \\
\hline None & $146(89.0)$ & $68(90.7)$ & 69 (86.3) & $9(100)$ \\
\hline BP-lowering (urapidil, clonidine) & $16(9.8)$ & $7(9.3)$ & $9(11.3)$ & $0(0)$ \\
\hline BP-increasing (sympathomimetics) & $2(1.2)$ & $0(0)$ & $2(2.5)$ & $0(0)$ \\
\hline Procedure mean SBP $(\mathrm{mm} \mathrm{Hg})$ & $128(69-192)$ & 126 (83-168) & 126 (67-192) & $142(106-158)$ \\
\hline Procedure mean MAP $(\mathrm{mm} \mathrm{Hg})$ & $91(43-125)$ & $92(56-117)$ & $89(43-125)$ & $96(66-112)$ \\
\hline \multicolumn{5}{|l|}{ Rates of BP excursions vs. ref. $(\mathrm{n} / 10 \mathrm{~min})^{+}$} \\
\hline $\mathrm{SBP}>120 \%$ & $1.09(0-9.26)$ & $1.21(0-9.26)$ & $0.93(0-8.41)$ & $1.63(0-8.19)$ \\
\hline MAP $>120 \%$ & $1.19(0-9.74)$ & $1.31(0-8.92)$ & $1.03(0-9.74)$ & $1.95(0-8.75)$ \\
\hline $\mathrm{SBP}<80 \%$ & $0.97(0-9.20)$ & $1.26(0-9.04)$ & $0.82(0-9.20)$ & $0.66(0-0.98)$ \\
\hline MAP $<80 \%$ & $0.79(0-8.91)$ & $1.08(0-8.91)$ & $0.66(0-8.22)$ & $0.11(0-0.11)$ \\
\hline Inotropes during procedure & 77 (46.9) & $33(44.0)$ & $38(47.5)$ & $6(66.7)$ \\
\hline
\end{tabular}


Table 1. Continued

\begin{tabular}{|c|c|c|c|c|}
\hline Characteristic & All patients & $m R S 0-2$ & mRS 3-6 & Missing $\mathrm{mRS}$ \\
\hline \multicolumn{5}{|l|}{ Leptomeningeal collaterals } \\
\hline Poor ( $<50 \%$ as on unaffected side) & $32(19.51)$ & $6(8.0)$ & $24(30.0)$ & $2(22.2)$ \\
\hline Good ( $\geq 50 \%$ as on unaffected side) & $132(80.5)$ & 69 (92.0) & $56(70.0)$ & 7 (77.8) \\
\hline Reperfusion success ( $\mathrm{TICl}$ class $2 \mathrm{~b}-3$ ) & $121(73.8)$ & $65(86.7)$ & $50(62.5)$ & $6(66.7)$ \\
\hline \multicolumn{5}{|l|}{ Control CT } \\
\hline No infarction, no hemorrhage & $18(11.0)$ & $11(14.7)$ & $6(7.5)$ & $1(11.1)$ \\
\hline Infarction only, no hemorrhage & $117(71.3)$ & $53(70.6)$ & $56(70.0)$ & $8(88.9)$ \\
\hline Hemorrhage visible & $29(17.7)$ & $11(14.7)$ & $18(22.5)$ & $0(0)$ \\
\hline Ischemic lesion volume $\left(\mathrm{cm}^{3}\right)$ & $30.7(0-518)$ & $10.9(0-466)$ & $102(0-518)$ & $9.1(0-127)$ \\
\hline Post-procedure statin use & $110(67.5)$ & $43(57.3)$ & $62(77.5)$ & $5(62.5)$ \\
\hline
\end{tabular}

Values are presented as median (interquartile range) or number (\%).

mRS, modified Rankin Scale; BP, blood pressure; MAP, mean arterial pressure; NIHSS, National Institutes of Health Stroke Scale; TOAST, Trial of Org 10172 in Acute Stroke Treatment classification; rtPA, recombinant human tissue plasminogen activator; SBP, systolic blood pressure; TICl, Thrombolysis in Cerebral Infarction; CT, computed tomography.

${ }^{*} \mathrm{mRS}$ score: favorable (score 0 to 2 ) or poor (score 3 to 6 ); ${ }^{+}$Geometric mean ratio (interquartile range).

dure SPB/MAP appeared comparable, while rates of excursions to $>120 \%$ or to $<80 \%$ of the reference appeared higher in patients with $\mathrm{mRS}$ 0-2. Similar proportions of patients received sympathomimetics during EVT (Table 1). Patients with mRS 0-2 more frequently had good collaterals, were more frequently successfully reperfused, their ILV was lower and visible hemorrhages were less frequent. They were less frequently post-stroke statin users, likely due to confounding by indication (Table 1).

Full, reduced and final models were fitted to the outcome (see Supplementary Table 5 for complete models). Independent variables consistently not associated with $\mathrm{mRS}$ were consecutively removed (Figure 2). Final models are shown in Table 3. The rate of SBP/MAP excursions to $<80 \%$ of the reference was not associated with the outcome (Table 3). When not accounting for ILV, higher rate of SBP/MAP excursions to $>120 \%$, TICI grade 2b-3, good collaterals and pre-index stroke statin use were associated with mRS 0-2 (Table 3, Model A). Higher onadmission NIHSS score, cardioembolic/unknown stroke etiology, post-index stroke statin use and older age were associated with lower odds of mRS 0-2 (Table 3, Model A). When accounted for, higher ILV was associated with lower odds of mRS 0-2 (Table 3, Model B). At the same time, the effects of SBP/ MAP excursions, $\mathrm{TICl}$ grade and of collaterals were reduced to include unity (Table 3, Model B). Mediation analysis (Figure 4) showed an indirect (via ILV) association between higher rates of SBP/MAP excursions to $>120 \%$ and mRS 0-2 (the same was shown for $\mathrm{TICl} 2 \mathrm{~b}-3$ and good collaterals).

Several models (Supplementary Table 5) indicated an interaction between the rate of SBP/MAP excursions to $>120 \%$ and $\mathrm{TICl}$ grade: (1) a strong association between higher rates and
mRS 0-2 in patients with $\mathrm{TICl} 2 b-3(n=115)$ and none in patients with TICl 0-2a ( $n=40)$ with point-estimates $<1.0$; (2) no association between $\mathrm{TICl} 2 \mathrm{~b}-3$ and $\mathrm{mRS} 0-2$ in patients with zero excursion rates, with clear-cut associations between $\mathrm{TICl} 2 \mathrm{~b}-3$ and higher odds of mRS 0-2 at rates around 1.0/10 minutes and higher (Supplementary Table 5, Models final C, D). We consider final models without the interaction (Table 3 ) to more generally describe the data than the models with this (uncertain) interaction that did not indicate harms ascribable to SPB/MAP excursions (for the full rationale, see Supplementary Methods).

\section{Discussion}

We found that in anterior circulation stroke patients treated with EVT/rtPA, in whom BP was generally kept within the recommended limits, higher in-procedure SBP/MAP was associated with a better 3-month functional outcome. The findings are supported by the association of higher rates of in-procedure SBP/MAP excursions to $>120 \%$ of the reference values, and of higher reference BP with mRS 0-2. The fact that no such association was observed for (higher) in-procedure mean BP is likely due to aliasing by the effects of a higher number of excursions over a high(er) reference value. A stronger association between BP excursions and in-procedure mean BP at higher reference BP supports such a view. Data also suggest associations between a higher rate of SBP/MAP excursions to $>120 \%$ and lower ILV. Higher ILV was associated with lower odds of mRS 0-2, and mediation analysis that differentiates effects identifying indirect path ${ }^{21}$ plausible in a temporal and pathophysiological sense disclosed its mediator role: BP excursions 
Table 2. Relationship between BP/BP excursions during endovascular thrombectomy to values exceeding $\pm 20 \%$ of the reference BP and control computed tomography findings

\begin{tabular}{|c|c|c|c|c|}
\hline \multirow{2}{*}{ Variable } & \multicolumn{2}{|c|}{ Systolic blood pressure } & \multicolumn{2}{|c|}{ Mean arterial pressure } \\
\hline & GMR or OR $(95 \% \mathrm{Cl})$ & $P$ & GMR or OR $(95 \% \mathrm{Cl})$ & $P$ \\
\hline \multicolumn{5}{|l|}{ Ischemic lesion volume } \\
\hline \multicolumn{5}{|l|}{ Default independent variables ${ }^{t, t}$} \\
\hline Rate of $\mathrm{BP}>120 \%$ of reference (by $1 / 10 \mathrm{~min}$ ) & $0.72(0.60-0.87)$ & $<0.001$ & $0.75(0.63-0.90)$ & 0.002 \\
\hline Rate of $\mathrm{BP}<80 \%$ of reference (by $1 / 10 \mathrm{~min}$ ) & $1.06(0.91-1.25)$ & 0.443 & $1.10(0.93-1.31)$ & 0.265 \\
\hline Reference BP (by $10 \mathrm{~mm} \mathrm{Hg}$ ) & $1.01(0.85-1.19)$ & 0.911 & $0.86(0.69-1.07)$ & 0.181 \\
\hline Rate of BP excursions $>120 \% *$ reference BP & $0.86(0.78-0.96)$ & 0.006 & $0.95(0.86-1.04)$ & 0.264 \\
\hline On-admission NIHSS score (by 1 point) & $1.06(1.01-1.11)$ & 0.014 & $1.06(1.01-1.10)$ & 0.021 \\
\hline $\mathrm{TICl}$ grade $2 \mathrm{~b}-3$ (vs. $0-2 \mathrm{a})$ & $0.36(0.20-0.67)$ & 0.001 & $0.36(0.20-0.67)$ & 0.042 \\
\hline Affected vessel: MCA1 vs. other & $0.65(0.36-1.17)$ & 0.153 & $0.72(0.40-1.29)$ & 0.270 \\
\hline Collaterals $\geq 50 \%$ as unaffected side (vs. no) & $0.33(0.16-0.68)$ & 0.002 & $0.33(0.16-0.67)$ & 0.003 \\
\hline \multicolumn{5}{|l|}{ Selected independent variables } \\
\hline Men (vs. women) & $1.84(1.07-3.17)$ & 0.028 & $1.78(1.03-3.07)$ & 0.038 \\
\hline \multicolumn{5}{|l|}{ Post-procedure visible hemorrhages ${ }^{t, \S}$} \\
\hline \multicolumn{5}{|l|}{ Default independent variables } \\
\hline Rate of $\mathrm{BP}>120 \%$ of reference (by $1 / 10 \mathrm{~min}$ ) & $1.03(0.72-1.48)$ & 0.855 & $1.10(0.82-1.50)$ & 0.479 \\
\hline Rate of $\mathrm{BP}<80 \%$ of reference (by $1 / 10 \mathrm{~min}$ ) & $0.80(0.58-1.11)$ & 0.183 & $0.77(0.53-1.12)$ & 0.178 \\
\hline Reference BP (by $10 \mathrm{~mm} \mathrm{Hg}$ ) & $1.48(0.99-2.28)$ & 0.056 & $1.86(1.00-3.47)$ & 0.051 \\
\hline Procedure mean BP (by $10 \mathrm{~mm} \mathrm{Hg}$ ) & $0.65(0.40-0.96)$ & 0.032 & $0.52(0.26-0.95)$ & 0.034 \\
\hline $\mathrm{TICl}$ grade $2 \mathrm{~b}-3$ (vs. $0-2 \mathrm{a}$ ) & $0.62(0.25-1.53)$ & 0.298 & $0.61(0.25-1.50)$ & 0.280 \\
\hline \multicolumn{5}{|l|}{ Selected independent variables } \\
\hline Cardioembolic/unknown etiology (vs. LAA) & $3.61(0.81-28.8)$ & 0.095 & $3.51(0.64-19.4)$ & 0.149 \\
\hline History of coronary heart disease & $2.58(1.04-6.42)$ & 0.041 & $2.56(1.03-6.38)$ & 0.043 \\
\hline
\end{tabular}

$\mathrm{BP}$, blood pressure; GMR, geometric mean ratio; $\mathrm{OR}$, odds ratio; $\mathrm{Cl}$, confidence interval; NIHSS, National Institutes of Health Stroke Scale; $\mathrm{TICl}$, Thrombolysis in Cerebral Infarction; MCA, middle cerebral artery (segment 1 or 2); LAA, large artery atherosclerosis.

${ }^{*}$ The asterisk is a part of the interaction term; ${ }^{+}$Reduced models; ${ }^{*} \mathrm{GMR}(95 \% \mathrm{Cl}) ;{ }^{5} \mathrm{OR}(95 \% \mathrm{Cl})$.

are directly associated with lower ILV, lower ILV is directly associated with a better functional outcome, excursions affect functional outcome "through" the effects on ILV.

The analysis has limitations common to similar recent reports on the topic ${ }^{13-16}$-it did not account for the post-procedural $\mathrm{BP}$; it did not test specific BP targets but evaluated spontaneously occurring patterns, where some, otherwise possible patterns, might not have occurred. Also, data on the initial infarct volume were not available. This leaves space for the assumption that patients with a larger initial infarct volume might have been managed at lower BP levels (to avoid hemorrhagic transformation), while a less stringent BP management might have been in place in patients with lower volumes, thus spuriously "grouping" poorer outcomes and lower BP (large initial volume), and better outcomes and higher BP (smaller initial volume). However, all observed associations were adjusted for on-admission NIHSS and use of antihypertensives between admission and EVT. It is reasonable to consider that these two variables to a large extent subsumed the "impact" of the initial infarct volume: if higher on-admission NIHSS (a "proxy" for larger infarct volume) would "go" together with higher on-admission BP, it would be associated also with immediate use of antihypertensives. However, our data showed that (1) higher on-admission BP but not NIHSS was associated with antihypertensive use; (2) BP reduction from admission to EVT (adjusted for on-admission BP) was similar in patients treated and not treated with antihypertensives; (3) neither on-admission NIHSS nor the use of antihypertensives were associated with reference BP or with in-procedure mean BP. Taken together, the findings suggest that the observed BP patterns were likely a consequence of the intended management within the recommended limits, and were not driven by the initial stroke severity (i.e., initial infarct volume). Furthermore, we consider invasive frequent BP recordings, inclusion of unselected patients, uniform anesthesia, and accounting for a number of confounders to be the study strengths. 
Table 3. Relationship between BP/BP excursions during endovascular thrombectomy to values exceeding $\pm 20 \%$ of the reference value and 3-month modified Rankin Scale score 0-2 (final models)

\begin{tabular}{|c|c|c|c|c|}
\hline \multirow{2}{*}{ Variable } & \multicolumn{2}{|c|}{ Systolic blood pressure } & \multicolumn{2}{|c|}{ Mean arterial pressure } \\
\hline & OR $(95 \% \mathrm{Cl})$ & $P$ & OR $(95 \% \mathrm{Cl})$ & $P$ \\
\hline \multicolumn{5}{|l|}{ Model A: without imaging outcomes } \\
\hline \multicolumn{5}{|l|}{ Default independents } \\
\hline Rate of BP $>120 \%$ of reference (by $1 / 10 \mathrm{~min}$ ) & $1.33(1.01-1.76)$ & 0.038 & $1.30(1.01-1.66)$ & 0.029 \\
\hline Rate of $\mathrm{BP}<80 \%$ of reference (by $1 / 10 \mathrm{~min}$ ) & $0.85(0.67-1.08)$ & 0.174 & $0.85(0.65-1.10)$ & 0.218 \\
\hline Reference BP (by $10 \mathrm{~mm} \mathrm{Hg}$ ) & $1.37(1.06-1.77)$ & 0.013 & $1.49(1.06-2.08)$ & 0.016 \\
\hline On-admission NIHSS score (by 1 point) & $0.85(0.78-0.93)$ & $<0.001$ & $0.86(0.80-0.94)$ & $<0.001$ \\
\hline $\mathrm{TICl}$ grade $2 \mathrm{~b}-3$ (vs. $0-2 \mathrm{a})$ & $3.96(1.34-11.7)$ & 0.009 & $3.72(1.26-11.0)$ & 0.013 \\
\hline Collaterals $\geq 50 \%$ as unaffected side (vs. no) & $4.62(1.45-11.2)$ & 0.007 & $4.71(1.44-15.4)$ & 0.006 \\
\hline \multicolumn{5}{|l|}{ Selected independents } \\
\hline Cardioembolic/unknown etiology (vs. LAA) & $0.25(0.07-090)$ & 0.026 & $0.22(0.06-0.84)$ & 0.018 \\
\hline Pre-index stroke statin use & $5.88(1.55-22.3)$ & 0.005 & $4.71(1.27-17.5)$ & 0.013 \\
\hline Post-index stroke statin use & $0.32(0.12-0.82)$ & 0.015 & $0.34(0.13-0.87)$ & 0.020 \\
\hline Age (by 10 yr) & $0.69(0.50-0.96)$ & 0.020 & $0.72(0.52-0.99)$ & 0.039 \\
\hline \multicolumn{5}{|l|}{ Model B: including ischemic lesion volume } \\
\hline \multicolumn{5}{|l|}{ Default independents } \\
\hline Rate of BP $>120 \%$ of reference (by $1 / 10 \mathrm{~min}$ ) & $1.25(0.95-1.71)$ & 0.114 & $1.22(0.95-1.61)$ & 0.115 \\
\hline Rate of $\mathrm{BP}<80 \%$ of reference (by $1 / 10 \mathrm{~min}$ ) & $0.88(0.69-1.11)$ & 0.291 & $0.89(0.67-1.15)$ & 0.371 \\
\hline Reference BP (by 10 mm Hg) & $1.33(1.02-1.77)$ & 0.034 & $1.37(0.97-1.97)$ & 0.071 \\
\hline On-admission NIHSS score (by 1 point) & $0.87(0.79-0.95)$ & 0.002 & $0.88(0.81-0.96)$ & 0.003 \\
\hline $\mathrm{TICl}$ grade $2 \mathrm{~b}-3$ (vs. $0-2 \mathrm{a})$ & $2.73(0.89-9.10)$ & 0.079 & $2.55(0.83-8.46)$ & 0.101 \\
\hline Collaterals $\geq 50 \%$ as unaffected side (vs. no) & $2.95(0.88-11.2)$ & 0.080 & $3.04(0.91-11.5)$ & 0.071 \\
\hline Ischemic lesion volume (by 2.718 -fold) & $0.65(0.50-0.83)$ & $<0.001$ & $0.66(0.50-0.84)$ & $<0.001$ \\
\hline \multicolumn{5}{|l|}{ Selected independents } \\
\hline Cardioembolic/unknown etiology (vs. LAA) & $0.19(0.05-0.69)$ & 0.011 & $0.16(0.04-0.62)$ & 0.007 \\
\hline Pre-index stroke statin use & $3.94(1.08-16.8)$ & 0.037 & $3.48(0.97-14.8)$ & 0.057 \\
\hline Post-index stroke statin use & $0.34(0.12-0.88)$ & 0.026 & $0.33(0.12-0.87)$ & 0.025 \\
\hline Age (by 10 yr) & $0.63(0.43-0.87)$ & 0.005 & $0.65(0.45-0.90)$ & 0.010 \\
\hline
\end{tabular}

$\mathrm{BP}$, blood pressure; OR, odds ratio; Cl, confidence interval; NIHSS, National Institutes of Health Stroke Scale; TICl, Thrombolysis in Cerebral Infarction; LAA, large artery atherosclerosis.

Present findings apparently disagree with a similar recent report..$^{13}$ Individual in-procedure BP indicators (maximum, minimum, dips) were evaluated for association with 30-day mRS in 147 EVT-treated patients. In multivariate models (13 independents), higher maximum SBP was associated with lower odds of mRS 0-2. The only other significant effect was admission NIHSS, while collaterals, stroke etiology, ILV were not considered. ${ }^{13}$ The cohort differed from ours in respect to anesthesia (46\% GA), less common rtPA use (35.8\%), fewer reperfusion successes (50\%), lower mRS 0-2 achievement (17\%), and fewer BP recordings (every 5 minutes). ${ }^{13}$ The apparently discordant results might be due to these methodological and cohort differences.
Three further similar studies indirectly support the present findings but also oppose them in a way. ${ }^{14-16}$ All three studies suggest benefits of "higher" in-procedure BP, but imply this indirectly by recognizing detrimental consequences of low(er) MAP, i.e., in-procedure MAP dips, which we could not confirm. In one study (EVT under GA), at least one episode of MAP decline $>40 \%$ from the baseline value was associated with 3-month mRS 3-6, but time spent at this MAP level was not. ${ }^{14}$ Another (smaller) analysis (EVT under GA) reported no association between the largest in-procedure MAP decline from the baseline value and 3-month mRS. However, lower (vs. baseline) average in-procedure MAP was associated with poorer mRS, while higher average MAP was associated with better $\mathrm{mRS}_{1}{ }_{1}^{15}$ 

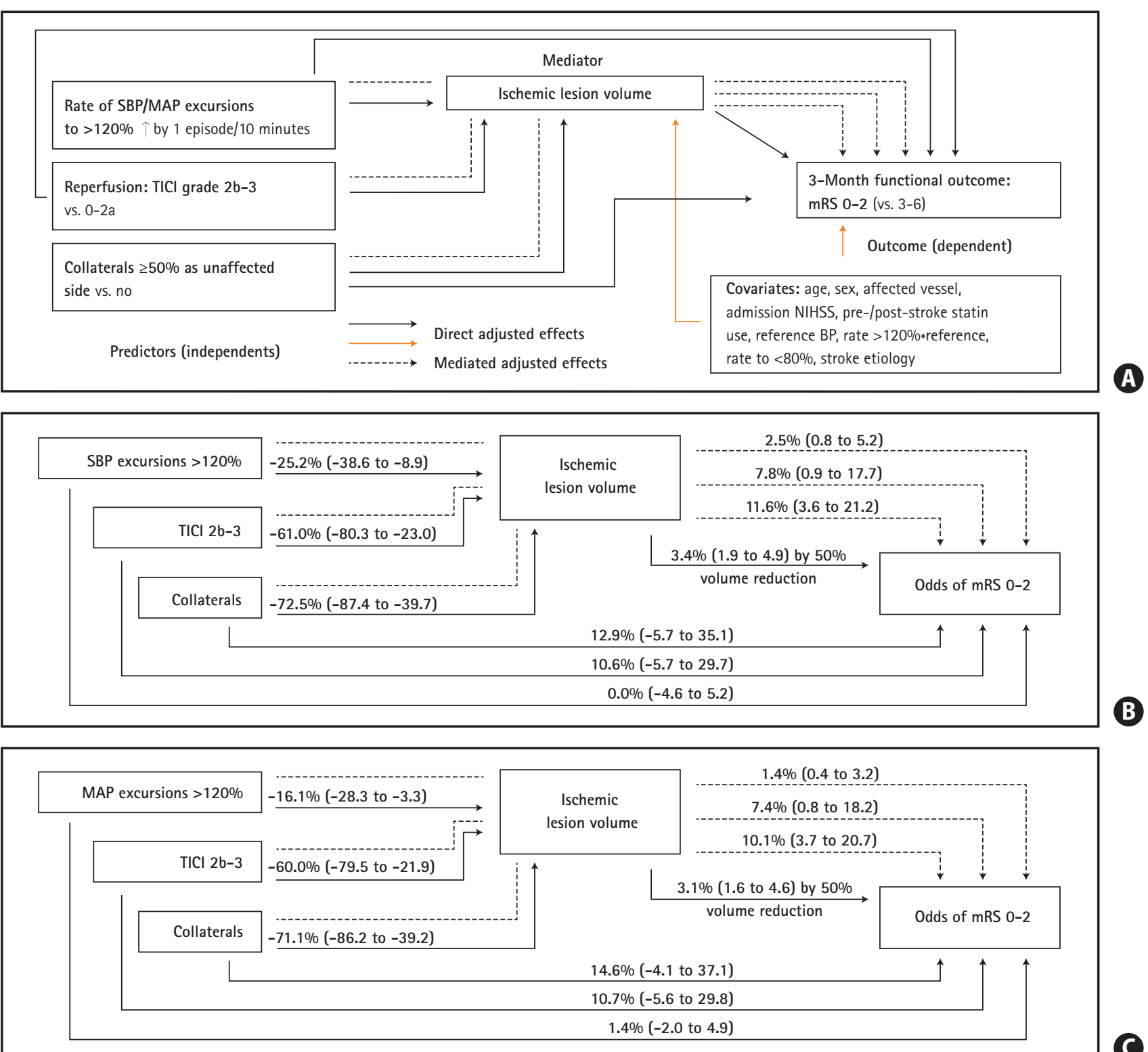

Figure 4. Mediation analysis: association of in-procedure systolic blood pressure (SBP) and mean arterial pressure (MAP) excursions to $>120 \%$ of their reference values with 3-month modified Rankin Scale (mRS) score 0-2 is mediated through their association with the ischemic lesion volume (ILV). (A) Outline of associations. All associations are adjusted for all other model effects. Effects are from models analyzing ILV (Table 2) and mRS (Table 3). (B) Mediation model for SBP. (C) Mediation model for MAP. Higher rate of blood pressure (BP) excursions, Thrombolysis in Cerebral Infarction (TICl) scale grade $2 \mathrm{~b}-3$ and good collaterals are each directly associated with lower ILV; lower ILV is directly associated with higher odds of mRS 0-2; direct association of these predictors with mRS 0-2 is uncertain; each is associated with mRS 0-2 indirectly, via ILV. Effects are expressed as percent change in ILV or odds of mRS 0-2 with 95\% confidence interval. NIHSS, National Institute of Stroke Scale.

thus being in line with present results. Detrimental effect of low(er) in-procedure MAP was suggested also in EVT under conscious sedation-lowest in-procedure MAP $<100 \mathrm{~mm} \mathrm{Hg}$ was associated with 3-month mRS 3-6. ${ }^{16}$

Despite methodological differences between the present and previous reports, there is evidence of detrimental consequences of low in-procedure MAP. ${ }^{14-16}$ This is in apparent disagreement with the reported association between high(er)
SBP during the first 24 hours post-EVT and poor functional outcomes. ${ }^{9,22}$ It seems that management of BP in anterior circulation acute stroke patients undergoing EVT requires different strategies: one focused on in-procedure targets and prevention of excessive dips, the other focused on the post-procedural period targeting lower levels with a prompt management of excessive peaks. 


\section{Conclusions}

In the EVT-treated acute anterior circulation stroke patients in whom BP is managed within the currently recommended limits, a better functional outcome might be achieved by targeting in-procedure BP that exceeds the pre-procedure values by more than $20 \%$.

\section{Supplementary materials}

Supplementary materials related to this article can be found online at https://doi.org/10.5853/jos.2018.01305.

\section{Disclosure}

The authors have no financial conflicts of interest.

\section{References}

1. National Institute of Neurological Disorders and Stroke rt-PA Stroke Study Group. Tissue plasminogen activator for acute ischemic stroke. N Engl J Med 1995;333:1581-1587.

2. Hacke $W$, Kaste $M$, Bluhmki $E$, Brozman $M$, Dávalos $A_{1}$ Guidetti $D_{1}$ et al. Thrombolysis with alteplase 3 to 4.5 hours after acute ischemic stroke. N Engl J Med 2008;359:13171329.

3. Berkhemer OA, Fransen PS, Beumer D, van den Berg LA, Lingsma $\mathrm{HF}_{\text {, }}$ Yoo AJ, et al. A randomized trial of intraarterial treatment for acute ischemic stroke. N Engl J Med 2015;372:11-20.

4. Powers WJ, Rabinstein AA, Ackerson T, Adeoye OM, Bambakidis NC, Becker K, et al. 2018 Guidelines for the early management of patients with acute ischemic stroke: a guideline for healthcare professionals from the American Heart Association/ American Stroke Association. Stroke 2018;49:e46-e110.

5. Strandgaard S. Autoregulation of cerebral blood flow in hypertensive patients. The modifying influence of prolonged antihypertensive treatment on the tolerance to acute, druginduced hypotension. Circulation 1976;53:720-727.

6. Strandgaard S, Olesen J, Skinhoj E, Lassen NA. Autoregulation of brain circulation in severe arterial hypertension. $\mathrm{Br}$ Med J 1973;1:507-510.

7. Dirnagl U, Pulsinelli W. Autoregulation of cerebral blood flow in experimental focal brain ischemia. J Cereb Blood Flow Metab 1990;10:327-336.

8. Mistry EA, Mistry AM, Nakawah MO, Khattar NK, Fortuny $E M$, Cruz AS, et al. Systolic blood pressure within 24 hours after thrombectomy for acute ischemic stroke correlates with outcome. J Am Heart Assoc 2017;6:e066167.
9. Bennett AE, Wilder MJ, McNally JS, Wold JJ, Stoddard GJ, Majersik JJ, et al. Increased blood pressure variability after endovascular thrombectomy for acute stroke is associated with worse clinical outcome. J Neurointerv Surg 2018;10:823-827.

10. Castillo J, Leira R, García MM, Serena J, Blanco M, Dávalos $A$. Blood pressure decrease during the acute phase of ischemic stroke is associated with brain injury and poor stroke outcome. Stroke 2004;35:520-526.

11. Grabska K, Niewada M, Sarzyńska-Długosz I, Kamiński B, Członkowska A. Pulse pressure: independent predictor of poor early outcome and mortality following ischemic stroke. Cerebrovasc Dis 2009;27:187-192.

12. Leonardi-Bee J, Bath PM, Phillips SJ, Sandercock PA; IST Collaborative Group. Blood pressure and clinical outcomes in the International Stroke Trial. Stroke 2002;33:1315-1320.

13. John $S$, Hazaa W, Uchino $K$, Toth $G$, Bain $M$, Thebo $U$, et al. Lower intraprocedural systolic blood pressure predicts good outcome in patients undergoing endovascular therapy for acute ischemic stroke. Interv Neurol 2016;4:151-157.

14. Löwhagen Hendén $P$, Rentzos $A$, Karlsson JE, Rosengren $L$, Sundeman $H_{1}$ Reinsfelt $B$, et al. Hypotension during endovascular treatment of ischemic stroke is a risk factor for poor neurological outcome. Stroke 2015;46:2678-2680.

15. Treurniet KM, Berkhemer OA, Immink RV, Lingsma HF, Wardvan der Stam VMC, Hollmann MW, et al. A decrease in blood pressure is associated with unfavorable outcome in patients undergoing thrombectomy under general anesthesia. J Neurointerv Surg 2018;10:107-111.

16. Whalin MK, Halenda KM, Haussen DC, Rebello LC, Frankel $M R$, Gershon RY, et al. Even small decreases in blood pressure during conscious sedation affect clinical outcome after stroke thrombectomy: an analysis of hemodynamic thresholds. AJNR Am J Neuroradiol 2017;38:294-298.

17. Wahlgren $N$, Ahmed N, Dávalos $A$, Ford GA, Grond M, Hacke $W$, et al. Thrombolysis with alteplase for acute ischaemic stroke in the Safe Implementation of Thrombolysis in Stroke-Monitoring Study (SITS-MOST): an observational study. Lancet 2007;369:275-282.

18. Adams HP Jr, Bendixen BH, Kappelle ப, Biller J, Love BB, Gordon DL, et al. Classification of subtype of acute ischemic stroke. Definitions for use in a multicenter clinical trial. TOAST. Trial of Org 10172 in Acute Stroke Treatment. Stroke 1993;24:35-41.

19. Pikija S, Trkulja V, Mutzenbach JS, McCoy MR, Ganger $P$, Sellner J. Fibrinogen consumption is related to intracranial clot burden in acute ischemic stroke: a retrospective hyperdense artery study. J Trans/ Med 2016;14:250.

20. John S, Hazaa W, Uchino K, Hussain MS. Timeline of blood 
pressure changes after intra-arterial therapy for acute ischemic stroke based on recanalization status. J Neurointerv Surg 2017;9:455-458.

21. Preacher KJ, Hayes AF. SPSS and SAS procedures for estimating indirect effects in simple mediation models. Behav Res
Methods Instrum Comput 2004;36:717-731.

22. Goyal N, Tsivgoulis G, Pandhi A, Chang JJ, Dillard K, Ishfaq $M F$, et al. Blood pressure levels post mechanical thrombectomy and outcomes in large vessel occlusion strokes. Neurology 2017;89:540-547. 
Supplementary Table 1. Routine laboratory test results taken on admission or within 24 hours, overall and by mRS score at 3 months

\begin{tabular}{lcccc}
\hline Variable & All patients & mRS 0-2 & mRS 3-6 & Missing mRS \\
\hline Number & 164 & 75 & 80 & 9 \\
Serum creatinine $(\mu \mathrm{mol} / \mathrm{L})$ & $77(16-249)$ & $76(40-126)$ & $85(16-249)$ & $73(50-93)$ \\
Serum glucose $(\mathrm{mmol} / \mathrm{L})$ & $6.6(4.5-15.5)$ & $6.2(4.5-10.3)$ & $7.1(5.3-15.5)$ & $6.4(4.8-9.7)$ \\
Total cholesterol $(\mathrm{mmol} / \mathrm{L})$ & $4.1(2.0-7.8)$ & $4.3(2.5-6.4)$ & $3.7(2.0-7.8)$ & $4.7(2.8-5.2)$ \\
HDL-C $(\mathrm{mmol} / \mathrm{L})$ & $1.1(0.31-2.8)$ & $1.1(0.47-2.4)$ & $1.1(0.31-2.2)$ & $1.7(0.80-2.3)$ \\
LDL-C $(\mathrm{mmol} / \mathrm{L})$ & $2.4(0.52-4.7)$ & $2.8(0.59-4.5)$ & $2.1(0.52-4.7)$ & $2.2(1.7-3.9)$ \\
Triglycerides $(\mathrm{mmol} / \mathrm{L})$ & $1.17(0.34-4.55)$ & $1.12(0.46-4.55)$ & $1.23(0.34-3.23)$ & $1.16(0.56-2.08)$ \\
Fibrinogen $(\mu \mathrm{mol} / \mathrm{L})$ & $9.9(2.9-23.1)$ & $9.9(3.7-17.0)$ & $10.0(2.9-23.1)$ & $10.3(7.5-11.6)$ \\
C-reactive protein $(\mathrm{mg} / \mathrm{L})$ & $0.36(0.01-9.85)$ & $0.29(0.01-8.65)$ & $0.46(0.04-9.85)$ & $0.38(0.07-1.79)$ \\
Red blood cells $\left(\times 10^{12} / \mathrm{L}\right)$ & $4.4(2.8-5.7)$ & $4.5(3.4-5.4)$ & $4.2(2.8-5.7)$ & $4.6(3.9-5.1)$ \\
Hematocrit $(\%)$ & $39.1(24.8-52.1)$ & $39.5(24.9-46.4)$ & $37.9(24.6-52.1)$ & $41.1(35.4-45.6)$ \\
Platelets $\left(\times 10^{9} / \mathrm{L}\right)$ & $220(67-572)$ & $229(67-572)$ & $212(101-431)$ & $224(174-286)$ \\
White blood cells $\left(\times 10^{9} / \mathrm{L}\right)$ & $8.1(3.0-70.9)$ & $8.2(3.0-70.9)$ & $8.4(3.2-27.2)$ & $7.4(6.0-10.1)$ \\
\hline
\end{tabular}

Values are presented as median (interquartile range).

mRS, modified Rankin Scale; HDL-C, high density lipoprotein cholesterol; LDL-C, low density lipoprotein cholesterol. 
Supplementary Table 2. Absolute (minutes) and relative (\%) time spent with SBP and MAP $>120 \%$ or $<80 \%$ of the reference value, overall and by mRS score at 3 months

\begin{tabular}{lcccc}
\hline Variable & All patients & mRS 0-2 & mRS 3-6 & Missing mRS \\
\hline Number & 164 & 75 & 80 & 9 \\
SBP $>120 \%$ of reference $(\mathrm{min})$ & $8.6(0-158)$ & $8.6(0-84)$ & $8.6(0-158)$ & $7.3(0-59)$ \\
MAP $>120 \%$ of reference $(\mathrm{min})$ & $9.5(0-223)$ & $9.2(0-127)$ & $9.7(0-223)$ & $10.7(0-63)$ \\
SBP $<80 \%$ of reference $(\mathrm{min})$ & $8.9(0-152)$ & $9.0(0-143)$ & $8.9(0-152)$ & $7.6(0-9)$ \\
MAP $<80 \%$ of reference $(\mathrm{min})$ & $7.2(0-160)$ & $7.8(0-75)$ & $7.3(0-160)$ & $1(0-1)$ \\
SBP $>120 \%$ of reference $(\%)$ & $10.9(0-93)$ & $12.1(0-93)$ & $9.3(0-84)$ & $16.3(0-82)$ \\
MAP $>120 \%$ of reference $(\%)$ & $11.9(0-97)$ & $13.1(0-89)$ & $10.3(0-97)$ & $19.5(0-88)$ \\
SBP $<80 \%$ of reference $(\%)$ & $9.6(0-92)$ & $12.3(0-90)$ & $8.2(0-92)$ & $6.6(0-9.6)$ \\
MAP $<80 \%$ of reference $(\%)$ & $7.8(0-89)$ & $10.6(0-89)$ & $6.6(0-82)$ & $1(0-1)$ \\
\hline
\end{tabular}

Values are presented as geometric mean (range).

SBP, systolic blood pressure; MAP, mean arterial pressure; mRS, modified Rankin Scale. 
Supplementary Table 3. Relationship between BP/BP excursions during EVT to values exceeding $\pm 20 \%$ of the reference value and ischemic lesion volume (computed tomography scans)

\begin{tabular}{|c|c|c|c|c|}
\hline \multirow{2}{*}{ Model } & \multicolumn{2}{|c|}{ Systolic blood pressure } & \multicolumn{2}{|c|}{ Mean arterial pressure } \\
\hline & GMR (95\% Cl) & $P$ & GMR (95\% Cl) & $P$ \\
\hline \multicolumn{5}{|l|}{ Full A: no interactions } \\
\hline \multicolumn{5}{|l|}{ Default independent variables } \\
\hline Rate of $\mathrm{BP}>120 \%$ of reference (by $1 / 10 \mathrm{~min}$ ) & $0.78(0.62-0.96)$ & 0.021 & $0.79(0.66-0.93)$ & 0.006 \\
\hline Rate of $\mathrm{BP}<80 \%$ of reference (by $1 / 10 \mathrm{~min}$ ) & $1.02(0.83-1.26)$ & 0.845 & $1.11(0.89-1.39)$ & 0.342 \\
\hline Reference BP (by 10 mm Hg) & $1.00(0.76-1.31)$ & 0.989 & $0.79(0.53-1.17)$ & 0.242 \\
\hline Procedure mean BP (by $10 \mathrm{~mm} \mathrm{Hg})^{+}$ & $0.92(0.70-1.20)$ & 0.538 & $1.06(0.71-1.57)$ & 0.772 \\
\hline Procedure duration (by $10 \mathrm{~min}$ ) & $1.01(0.95-1.08)$ & 0.764 & $1.02(0.95-1.08)$ & 0.639 \\
\hline BP measurements taken (by 10) & $1.07(0.98-1.17)$ & 0.119 & $1.08(0.99-1.17)$ & 0.098 \\
\hline On-admission NIHSS score (by 1 point) & $1.05(1.00-1.11)$ & 0.037 & $1.05(1.00-1.10)$ & 0.036 \\
\hline $\mathrm{TICl}$ grade $2 \mathrm{~b}-3$ (vs. $0-2 \mathrm{a})$ & $0.46(0.24-0.91)$ & 0.026 & $0.51(0.26-0.98)$ & 0.044 \\
\hline Affected vessel: MCA1 vs. MCA2 & $0.71(0.25-1.98)$ & 0.508 & $0.81(0.29-2.23)$ & 0.677 \\
\hline Affected vessel: MCA1 vs. tandem occlusion & $0.70(0.34-1.42)$ & 0.316 & $0.73(0.37-1.45)$ & 0.371 \\
\hline Collaterals $\geq 50 \%$ as unaffected side (vs. no) & $0.35(0.17-0.74)$ & 0.006 & $0.37(0.18-0.76)$ & 0.007 \\
\hline Antihypertensives before EVT (vs. no) & $1.41(0.55-3.56)$ & 0.471 & $1.30(0.53-3.23)$ & 0.565 \\
\hline \multicolumn{5}{|l|}{ Selected independent variables ${ }^{\ddagger}$} \\
\hline Men (vs. women) & $1.89(1.08-3.32)$ & 0.026 & $1.93(1.11-3.34)$ & 0.020 \\
\hline Model fit statistics (-2ResLL, AIC, BIC) & $682.44,712.44,757.60$ & & $674.54,704.54,749.70$ & \\
\hline \multicolumn{5}{|l|}{ Full B: with excursion*reference interactions } \\
\hline \multicolumn{5}{|l|}{ Default independent variables } \\
\hline Rate of $\mathrm{BP}>120 \%$ of reference (by $1 / 10 \mathrm{~min}$ ) & $0.73(0.57-0.92)$ & 0.008 & $0.74(0.58-0.93)$ & 0.011 \\
\hline Rate of $\mathrm{BP}<80 \%$ of reference (by $1 / 10 \mathrm{~min}$ ) & $1.11(0.81-1.52)$ & 0.500 & $1.16(0.87-1.54)$ & 0.317 \\
\hline Reference BP (by 10 mm Hg) & $0.88(0.66-1.16)$ & 0.358 & $0.75(0.49-1.14)$ & 0.178 \\
\hline Rate of BP excursions $>120 \% *$ reference BP & $0.86(0.77-0.96)$ & 0.008 & $0.96(0.87-1.06)$ & 0.404 \\
\hline Rate of BP excursions $<80 \% *$ reference BP & $0.98(0.92-1.04)$ & 0.492 & $0.98(0.91-1.07)$ & 0.710 \\
\hline Procedure mean BP (by $10 \mathrm{~mm} \mathrm{Hg})^{+}$ & $0.99(0.75-1.29)$ & 0.919 & $1.11(0.74-1.67)$ & 0.616 \\
\hline Procedure duration (by $10 \mathrm{~min}$ ) & $1.01(0.95-1.07)$ & 0.800 & $1.01(0.95-1.08)$ & 0.682 \\
\hline BP measurements taken (by 10 ) & $1.06(0.97-1.16)$ & 0.171 & $1.07(0.98-1.17)$ & 0.109 \\
\hline On-admission NIHSS score (by 1 point) & $1.05(1.00-1.10)$ & 0.041 & $1.05(1.00-1.10)$ & 0.052 \\
\hline $\mathrm{TICl}$ grade $2 \mathrm{~b}-3$ (vs. $0-2 \mathrm{a})$ & $0.47(0.24-0.92)$ & 0.027 & $0.51(0.26-0.99)$ & 0.046 \\
\hline Affected vessel: MCA1 vs. MCA2 & $0.86(0.31-2.40)$ & 0.874 & $0.85(0.30-2.37)$ & 0.748 \\
\hline Affected vessel: MCA1 vs. tandem occlusion & $0.61(0.30-1.24)$ & 0.170 & $0.73(0.36-1.45)$ & 0.367 \\
\hline Collaterals $\geq 50 \%$ as unaffected side (vs. no) & $0.37(0.18-0.77)$ & 0.008 & $0.37(0.18-0.76)$ & 0.007 \\
\hline Antihypertensives before EVT (vs. no) & $1.50(0.60-3.76)$ & 0.380 & $1.33(0.53-3.32)$ & 0.539 \\
\hline \multicolumn{5}{|l|}{ Selected independent variables ${ }^{*}$} \\
\hline Men (vs. women) & $1.88(1.08-3.29)$ & 0.026 & $1.91(1.09-3.34)$ & 0.024 \\
\hline Model fit statistics (-2ResLL, AIC, BIC) & $693.63,727.63,778.58$ & & $691.64,725.64,776.59$ & \\
\hline \multicolumn{5}{|l|}{ Full C: with excursions* $\mathrm{TICl}$ interactions } \\
\hline Rate of $\mathrm{BP}>120 \%$ of reference $* \mathrm{TICl}$ grade & $1.04(0.75-1.44)$ & 0.818 & $1.09(0.81-1.48)$ & 0.550 \\
\hline Rate of $\mathrm{BP}>120 \%$ at $\mathrm{TICl} 0-2 \mathrm{a}$ & $0.71(0.50-1.02)$ & - & $0.85(0.63-1.15)$ & - \\
\hline Rate of $\mathrm{BP}>120 \%$ at $\mathrm{TICl} 2 b-3$ & $0.73(0.57-0.94)$ & - & $0.77(0.65-0.93)$ & - \\
\hline Rate of $\mathrm{BP}<80 \%$ of reference $* \mathrm{TICl}$ grade & $0.87(0.64-1.18)$ & 0.365 & $0.87(0.61-1.25)$ & 0.456 \\
\hline Rate of $\mathrm{BP}<80 \%$ at $\mathrm{TICl} 0-2 \mathrm{a}$ & $0.95(0.70-1.27)$ & - & $1.00(0.70-1.42)$ & - \\
\hline Rate of $\mathrm{BP}<80 \%$ at $\mathrm{TICl} 2 \mathrm{~b}-3$ & $1.06(0.85-1.33)$ & - & $1.15(0.90-1.46)$ & - \\
\hline
\end{tabular}


Supplementary Table 3. Continued

\begin{tabular}{|c|c|c|c|c|}
\hline \multirow{2}{*}{ Model } & \multicolumn{2}{|c|}{ Systolic blood pressure } & \multicolumn{2}{|c|}{ Mean arterial pressure } \\
\hline & GMR $(95 \% \mathrm{Cl})$ & $P$ & GMR $(95 \% \mathrm{Cl})$ & $P$ \\
\hline $\mathrm{TICl}$ grade $2 \mathrm{~b}-3$ (vs. $0-2 \mathrm{a})$ & $0.45(0.23-0.90)$ & 0.023 & $0.50(0.25-0.96)$ & 0.039 \\
\hline Reference BP (by $10 \mathrm{~mm} \mathrm{Hg}$ ) & $1.01(0.77-1.32)$ & 0.961 & $0.80(0.54-1.20)$ & 0.284 \\
\hline $\begin{array}{l}\text { Procedure mean } \mathrm{BP} \text { (by } 10 \mathrm{~mm} \mathrm{Hg})^{+} \\
\text {(other effects were closely similar to } \mathrm{A} \text { and } \mathrm{B} \text { ) }\end{array}$ & $0.92(0.70-1.21)$ & 0.539 & $1.04(0.70-1.55)$ & 0.827 \\
\hline Model fit statistics (-2ResLL, AIC, BIC) & $685.14,719.14,770.09$ & & $676.94,710.94,761.89$ & \\
\hline \multicolumn{5}{|l|}{ Full D: with $\mathrm{BP} * \mathrm{TICl}$ interactions } \\
\hline Rate of $\mathrm{BP}>120 \%$ of reference (by $1 / 10 \mathrm{~min}$ ) & $0.72(0.57-0.92)$ & 0.009 & $0.79(0.66-0.94)$ & 0.007 \\
\hline Rate of $\mathrm{BP}<80 \%$ of reference (by $1 / 10 \mathrm{~min}$ ) & $1.00(0.83-1.27)$ & 0.809 & $1.11(0.88-1.39)$ & 0.372 \\
\hline $\mathrm{TICl}$ grade $2 \mathrm{~b}-3$ (vs. $0-2 \mathrm{a})$ & $0.48(0.25-0.94)$ & 0.033 & $0.51(0.26-1.00)$ & 0.050 \\
\hline Reference $\mathrm{BP} * \mathrm{TICl}$ grade & $0.92(0.67-1.26)$ & 0.619 & $0.91(0.56-1.47)$ & 0.704 \\
\hline Reference $\mathrm{BP}$ at $\mathrm{TICl} 0-2 \mathrm{a}$ & $0.95(0.68-1.33)$ & - & $0.75(0.46-1.22)$ & - \\
\hline Reference $\mathrm{BP}$ at $\mathrm{TICl} 2 \mathrm{~b}-3$ & $1.03(0.77-1.39)$ & - & $0.82(0.52-1.28)$ & - \\
\hline Procedure mean*BP TICl grade & $1.02(0.66-1.58)$ & 0.916 & $1.00(0.54-1.86)$ & 0.998 \\
\hline Procedure mean $\mathrm{BP}$ at $\mathrm{TICl} 0-2 \mathrm{a}$ & $0.93(0.61-1.41)$ & - & $1.05(0.59-1.86)$ & - \\
\hline $\begin{array}{l}\text { Procedure mean } \mathrm{BP} \text { at } \mathrm{TICl} 2 \mathrm{~b}-3 \\
\text { (other effects were closely similar to } \mathrm{A} \text { and } \mathrm{B} \text { ) }\end{array}$ & $0.91(0.67-1.22)$ & - & $1.05(0.67-1.63)$ & - \\
\hline Model fit statistics (-2ResLL, AIC, BIC) & $694.74,728.74,779.69$ & & $685.49,719.49,770.44$ & \\
\hline \multicolumn{5}{|l|}{ Reduced $^{\S}$} \\
\hline \multicolumn{5}{|l|}{ Default independent variables } \\
\hline Rate of $\mathrm{BP}>120 \%$ of reference (by $1 / 10 \mathrm{~min}$ ) & $0.72(0.60-0.87)$ & $<0.001$ & $0.75(0.63-0.90)$ & 0.002 \\
\hline Rate of $\mathrm{BP}<80 \%$ of reference (by $1 / 10 \mathrm{~min}$ ) & $1.06(0.91-1.25)$ & 0.443 & $1.10(0.93-1.31)$ & 0.265 \\
\hline Reference BP (by $10 \mathrm{~mm} \mathrm{Hg}$ ) & $1.01(0.85-1.19)$ & 0.911 & $0.86(0.69-1.07)$ & 0.181 \\
\hline Rate of BP excursions $>120 \% *$ reference BP & $0.86(0.78-0.96)$ & 0.006 & $0.95(0.86-1.04)$ & 0.264 \\
\hline On-admission NIHSS score (by 1 point) & $1.06(1.01-1.11)$ & 0.014 & $1.06(1.01-1.10)$ & 0.021 \\
\hline $\mathrm{TICl}$ grade $2 \mathrm{~b}-3$ (vs. $0-2 a$ ) & $0.36(0.20-0.67)$ & 0.001 & $0.36(0.20-0.67)$ & 0.042 \\
\hline Affected vessel: MCA1 vs. other & $0.65(0.36-1.17)$ & 0.153 & $0.72(0.40-1.29)$ & 0.270 \\
\hline Collaterals $\geq 50 \%$ as unaffected side (vs. no) & $0.33(0.16-0.68)$ & 0.002 & $0.33(0.16-0.67)$ & 0.003 \\
\hline \multicolumn{5}{|l|}{ Selected independent variables ${ }^{*}$} \\
\hline Men (vs. women) & $1.84(1.07-3.17)$ & 0.028 & $1.78(1.03-3.07)$ & 0.038 \\
\hline Model fit statistics (-2ResLL, AIC, BIC) & $666.00,688.00,721.41$ & & $666.27,688.27,721.68$ & \\
\hline
\end{tabular}

Two types of models (each separately for systolic and mean arterial pressure) were fitted: "full" (with different interaction terms) and "reduced" (effects based on biological and statistical plausibility). Effects are GMRs (lesion volume was In-transformed). GMRs for the interaction terms indicate whether the BP excursion effects were conditional on reference BP; or whether BP excursion effects and the effects of reference and procedure mean BP were conditional on the level of reperfusion ( $\mathrm{TICl}$ grade).

$\mathrm{BP}$, blood pressure; EVT, endovascular thrombectomy; GMR, geometric means ratio; Cl, confidence interval; NIHSS, National Institutes of Health Stroke Scale; $\mathrm{TICl}$, Thrombolysis in Cerebral Infarction; MCA, middle cerebral artery (segment 1 or 2); -2ResLL, 2 residual logarithmic likelihood; AIC, Akaike information criterion; BIC, Bayesian information criterion.

${ }^{*}$ The asterisk is a part of the interaction term; ${ }^{+}$Weighted mean as area under the BP-time curve divided by the time period covered; ${ }^{\ddagger}$ By stepwise selection procedure ( $P<0.200$ to enter/stay) among: age, sex, comorbidities, use of sympathomimetics during EVT, stroke etiology, time since symptom onset till the first vessel image, use of recombinant tissue plasminogen activator, pre-index stroke statin, antiplatelet or anticoagulant use; ${ }^{\circledR}$ Reduced model excluded interaction terms if no indication of moderation (for either systolic BP or mean arterial pressure), use of antihypertensives before EVT (highly insignificant and small number of treated patients), procedure mean BP (highly insignificant, determined by excursion rates, see Supplementary Analysis of Blood Pressure), procedure duration and number of BP measurements taken (highly insignificant). Affected vessel was dichotomized (MCA1/other). 
Supplementary Table 4. Relationship between BP/BP excursions during EVT to values exceeding $\pm 20 \%$ of the reference value and finding of hemorrhages on post-procedure computed tomography scans

\begin{tabular}{|c|c|c|c|c|}
\hline \multirow{2}{*}{ Model } & \multicolumn{2}{|c|}{ Systolic blood pressure } & \multicolumn{2}{|c|}{ Mean arterial pressure } \\
\hline & OR $(95 \% \mathrm{Cl})$ & $P$ & OR (95\% Cl) & $P$ \\
\hline
\end{tabular}

Full A: no interactions

Default independent variables

Rate of $B P>120 \%$ of reference (by $1 / 10 \mathrm{~min}$ )

Rate of BP $<80 \%$ of reference (by $1 / 10 \mathrm{~min}$ )

Reference BP (by $10 \mathrm{~mm} \mathrm{Hg}$ )

Procedure mean BP (by $10 \mathrm{~mm} \mathrm{Hg})^{+}$

Procedure duration (by $10 \mathrm{~min}$ )

BP measurements taken (by 10)

On-admission NIHSS score (by 1 point)

$\mathrm{TICl}$ grade $2 \mathrm{~b}-3$ (vs. 0-2a)

Use of rtPA (vs. no)

Collaterals $\geq 50 \%$ as unaffected side (vs. no)

Antihypertensives before EVT (vs. no)

$\begin{array}{ll}1.07(0.72-1.52) & 0.71 \\ 0.78(0.55-1.06) & 0.118 \\ 1.56(1.03-2.45) & 0.036 \\ 0.62(0.38-0.94) & 0.02 \\ 0.98(0.67-1.08) & 0.794 \\ 1.05(0.92-1.57) & 0.561 \\ 1.02(0.94-1.11) & 0.629 \\ 0.76(0.28-2.10) & 0.590 \\ 0.76(0.29-2.04) & 0.572 \\ 0.65(0.23-1.92) & 0.425 \\ 1.52(0.31-5.87) & 0.576 \\ 4.15(0.91-35.1) & 0.068 \\ 2.40(0.92-6.19) & 0.074\end{array}$

0.714

$1.14(0.82-1.57)$

$0.74(0.49-1.06)$

0.412

0.118

0.036

0.026

0.794

0.561

0.629

0.590

0.572

0.425

0.576

0.068

0.074

$-68.059,164.11,207.52$

Model fit statistics (LL, AIC, BIC)

Full B: with excursions*reference interactions

Default independent variables

Rate of $\mathrm{BP}>120 \%$ of reference (by $1 / 10 \mathrm{~min}$ )

Rate of BP $<80 \%$ of reference (by $1 / 10 \mathrm{~min}$ )

Reference BP (by $10 \mathrm{~mm} \mathrm{Hg}$ )

Rate of BP excursions > 120\%*reference BP

Rate of BP excursions $<80 \% *$ reference $\mathrm{BP}$

Procedure mean BP (by $10 \mathrm{~mm} \mathrm{Hg})^{+}$

Procedure duration (by $10 \mathrm{~min}$ )

BP measurements taken (by 10)

On-admission NIHSS score (by 1 point)

$\mathrm{TICl}$ grade $2 \mathrm{~b}-3$ (vs. $0-2 \mathrm{a}$ )

Use of rtPA (vs. no)

Collaterals $\geq 50 \%$ as unaffected side (vs. no)

Antihypertensives before EVT (vs. no)

Selected independent variables ${ }^{*}$

Cardioembolic/unknown etiology (vs. LAA)

History of coronary artery disease

Model fit statistics (LL, AIC, BIC)

Full C: with excursions* $\mathrm{TICl}$ interactions

Rate of $\mathrm{BP}>120 \%$ of reference $* \mathrm{TICl}$ grade

Rate of $\mathrm{BP}>120 \%$ at $\mathrm{TICl} 0-2 \mathrm{a}$

Rate of $\mathrm{BP}>120 \%$ at $\mathrm{TICl} 2 \mathrm{~b}-3$

Rate of $\mathrm{BP}<80 \%$ of reference $* \mathrm{TICl}$ grade

Rate of $\mathrm{BP}<80 \%$ at $\mathrm{TICl} 0-2 \mathrm{a}$

Rate of $\mathrm{BP}<80 \%$ at $\mathrm{TICl} 2 \mathrm{~b}-3$
$1.27(0.82-1.90)$

$0.94(0.55-1.53)$

$1.61(1.04-2.59)$

$1.20(0.94-1.64)$

$0.94(0.84-1.04)$

$0.56(0.33-0.87)$

$0.99(0.67-1.08)$

$1.06(0.92-1.59)$

1.03 (0.95-1.12)

$0.71(0.26-2.03)$

$0.85(0.32-2.35)$

$0.75(0.26-2.31)$

$1.28(0.25-5.16)$

$4.82(0.94-47.4)$

$2.45(0.93-6.42)$

$-66.167,164.33,213.93$

$1.69(0.82-5.34)$

$0.71(0.30-1.67)$

$1.21(0.82-1.77)$

$0.87(0.54-1.41)$

$0.86(0.57-1.31)$

$0.75(0.51-1.11)$
0.265

0.821

0.033

0.153

0.269

0.009

0.868

0.467

0.528

0.529

0.748

0.599

0.745

0.061

0.059

0.170

$-$

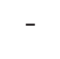

0.558

$-$

$-$
$2.00(1.05-4.10)$

0.101

0.034

0.030

0.814

0.585

0.567

0.560

0.609

0.355

0.539

$1.59(0.32-6.14)$

0.079

0.075

$2.40(0.91-6.22)$

$-68.578,165.15,208.55$

$\begin{array}{ll}1.29(0.81-1.98) & 0.265 \\ 0.97(0.59-1.49) & 0.900 \\ 2.31(1.19-4.87) & 0.014 \\ 1.05(0.87-1.32) & 0.641 \\ 0.86(0.63-1.02) & 0.088 \\ 0.46(0.21-0.90) & 0.023 \\ 0.99(0.65-1.08) & 0.832 \\ 1.06(0.92-1.61) & 0.474 \\ 1.03(0.95-1.12) & 0.489 \\ 0.76(0.28-2.08) & 0.583 \\ 0.85(0.33-2.34) & 0.748 \\ 0.66(0.23-1.98) & 0.444 \\ 1.66(0.32-6.71) & 0.512 \\ & \\ 5.31(1.04-53.3) & 0.045 \\ 2.70(1.01-7.21) & 0.036 \\ -66.931,165.86,215.46 & \end{array}$

$-66.931,165.86,215.46$

$\begin{array}{lc}1.41(0.77-3.70) & 0.303 \\ 0.84(0.40-1.80) & - \\ 1.19(0.86-1.65) & - \\ 0.61(0.25-1.13) & 0.122 \\ 0.96(0.60-1.52) & - \\ 0.59(0.32-1.09) & -\end{array}$


Supplementary Table 4. Continued

\begin{tabular}{|c|c|c|c|c|}
\hline \multirow{2}{*}{ Model } & \multicolumn{2}{|c|}{ Systolic blood pressure } & \multicolumn{2}{|c|}{ Mean arterial pressure } \\
\hline & OR $(95 \% \mathrm{Cl})$ & $P$ & OR $(95 \% \mathrm{Cl})$ & $P$ \\
\hline $\mathrm{TICl}$ grade $2 \mathrm{~b}-3$ (vs. 0-2a) & $0.97(0.32-2.95)$ & 0.958 & $0.89(0.30-2.64)$ & 0.828 \\
\hline Reference BP (by 10 mm Hg) & $1.51(0.99-2.40)$ & 0.053 & $1.91(0.99-3.97)$ & 0.052 \\
\hline $\begin{array}{l}\text { Procedure mean BP (by } 10 \mathrm{~mm} \mathrm{Hg})^{+} \\
\text {(other effects were closely similar to } \mathrm{A} \text { and } \mathrm{B} \text { ) }\end{array}$ & $0.63(0.38-0.95)$ & 0.029 & $0.53(0.25-0.99)$ & 0.048 \\
\hline Model fit statistics (LL, AIC, BIC) & $-66.756,165.51,215.11$ & & $-66.285,164.56,214.17$ & \\
\hline \multicolumn{5}{|l|}{ Full D: with $\mathrm{BP} * \mathrm{TICl}$ interactions } \\
\hline Rate of BP $>120 \%$ of reference (by $1 / 10 \mathrm{~min}$ ) & $1.06(0.71-1.51)$ & 0.763 & $1.09(0.78-1.52)$ & 0.582 \\
\hline Rate of $\mathrm{BP}<80 \%$ of reference (by $1 / 10 \mathrm{~min}$ ) & $0.79(0.55-1.09)$ & 0.159 & $0.77(0.49-1.11)$ & 0.172 \\
\hline $\mathrm{TICl}$ grade $2 \mathrm{~b}-3$ (vs. $0-2 \mathrm{a})$ & $0.87(0.31-2.45)$ & 0.799 & $0.91(0.32-2.58)$ & 0.853 \\
\hline Reference $\mathrm{BP} * \mathrm{TICl}$ grade & $0.77(0.45-1.26)$ & 0.296 & $0.62(0.26-1.37)$ & 0.241 \\
\hline Reference $\mathrm{BP}$ at $\mathrm{TICl} 0-2 \mathrm{a}$ & $1.78(1.07-2.95)$ & - & $2.45(1.07-5.60)$ & - \\
\hline Reference at $\mathrm{TICI} 2 b-3$ & $1.36(0.83-2.23)$ & - & $1.53(0.69-3.39)$ & - \\
\hline Procedure mean $\mathrm{BP} * \mathrm{TICl}$ grade & $1.28(0.66-2.64)$ & 0.465 & $2.20(0.79-7.32)$ & 0.136 \\
\hline Procedure mean $\mathrm{BP}$ at $\mathrm{TICl} 0-2 \mathrm{a}$ & $0.55(0.29-1.01)$ & - & $0.32(0.12-0.87)$ & - \\
\hline $\begin{array}{l}\text { Procedure mean } \mathrm{BP} \text { at } \mathrm{TICl} 2 \mathrm{~b}-3 \\
\text { (other effects were closely similar to } \mathrm{A} \text { and } \mathrm{B} \text { ) }\end{array}$ & $0.70(0.42-1.17)$ & - & $0.70(0.32-1.54)$ & - \\
\hline Model fit statistics (LL, AIC, BIC) & $-67.499,166.99,216.60$ & & $-67.439,166.88,216.48$ & \\
\hline \multicolumn{5}{|l|}{ Reduced $^{\S}$} \\
\hline \multicolumn{5}{|l|}{ Default independent variables } \\
\hline Rate of $B P>120 \%$ of reference (by $1 / 10 \mathrm{~min}$ ) & $1.03(0.72-1.48)$ & 0.855 & $1.10(0.82-1.50)$ & 0.479 \\
\hline Rate of $\mathrm{BP}<80 \%$ of reference (by $1 / 10 \mathrm{~min}$ ) & $0.80(0.58-1.11)$ & 0.183 & $0.77(0.53-1.12)$ & 0.178 \\
\hline Reference BP (by 10 mm Hg) & $1.48(0.99-2.28)$ & 0.056 & $1.86(1.00-3.47)$ & 0.051 \\
\hline Procedure mean BP (by $10 \mathrm{~mm} \mathrm{Hg})^{+}$ & $0.65(0.40-0.96)$ & 0.032 & $0.52(0.26-0.95)$ & 0.034 \\
\hline $\mathrm{TICl}$ grade $2 \mathrm{~b}-3$ (vs. $0-2 \mathrm{a})$ & $0.62(0.25-1.53)$ & 0.298 & $0.61(0.25-1.50)$ & 0.280 \\
\hline \multicolumn{5}{|l|}{ Selected independent variables ${ }^{*}$} \\
\hline Cardioembolic/unknown etiology (vs. LAA) & $3.61(0.81-28.8)$ & 0.095 & $3.51(0.64-19.4)$ & 0.149 \\
\hline History of coronary heart disease & $2.58(1.04-6.42)$ & 0.041 & $2.56(1.03-6.38)$ & 0.043 \\
\hline Model fit statistics (LL, AIC, BIC) & $-69.109,154.22,179.02$ & & $-69.792,155.58,180.38$ & \\
\hline
\end{tabular}

Two types of models (each separately for systolic and mean arterial pressure) were fitted: "full" (with different interaction terms) and "reduced" (effects based on biological and statistical plausibility). Effects are ORs. ORs for the interaction terms indicate whether the BP excursion effects were conditional on reference $\mathrm{BP}$; or whether BP excursion effects and the effects of reference and procedure mean BP were conditional on the level of reperfusion (TICl grade).

$\mathrm{BP}$, blood pressure; EVT, endovascular thrombectomy; OR, odds ratio; $\mathrm{Cl}$, confidence interval; NIHSS, National Institutes of Health Stroke Scale; TICl, Thrombolysis in Cerebral Infarction; rtPA, recombinant tissue plasminogen activator; LAA, large artery atherosclerosis; LL, logarithmic likelihood; AIC, Akaike information criterion; BIC, Bayesian information criterion.

${ }^{*}$ The asterisk is a part of the interaction term; ${ }^{+}$Weighted mean as area under the BP-time curve divided by the time period covered; ${ }^{*}$ By stepwise selection procedure $(P<0.200$ to enter/stay) among age, sex, comorbidities, use of sympathomimetics during EVT, stroke etiology, time since symptom onset till the first vessel image, type of affected vessel, pre-index stroke statin, antiplatelet or anticoagulant use; ${ }^{5}$ Reduced model excluded interaction terms (no indication of qualitative moderation), use of antihypertensives before EVT (small number of treated patients \& highly insignificant), existence of collaterals, use of rtPA, procedure duration and number of BP measurements taken since consistently highly insignificant. 
Supplementary Table 5. Relationship between BP/BP excursions during EVT to values exceeding $\pm 20 \%$ of the reference value and favorable functional outcome at 3 months (mRS, score $0-2$ )

\begin{tabular}{llll}
\hline \multirow{2}{*}{ Model } & \multicolumn{2}{c}{ Systolic blood pressure } & \multicolumn{2}{c}{ Mean arterial pressure } \\
\cline { 2 - 3 } & $\mathrm{OR}(95 \% \mathrm{Cl})$ & $P$ & $\mathrm{OR}(95 \% \mathrm{Cl})$ \\
\hline
\end{tabular}

Full A: without interactions

Default independent variables

Rate of $\mathrm{BP}>120 \%$ of reference (by $1 / 10 \mathrm{~min}$ )

Rate of BP $<80 \%$ of reference (by $1 / 10 \mathrm{~min}$ )

Reference BP (by $10 \mathrm{~mm} \mathrm{Hg}$ )

Procedure mean BP (by $10 \mathrm{~mm} \mathrm{Hg})^{+}$

Procedure duration (by $10 \mathrm{~min}$ )

$\mathrm{BP}$ measurements taken (by 10)

On-admission NIHSS score (by 1 point)

$\mathrm{TICl}$ grade 2b-3 (vs. 0-2a)

Collaterals $\geq 50 \%$ as unaffected side (vs. no)

Antihypertensive before EVT (vs. no)

Selected independent variables ${ }^{\dagger}$

Cardioembolic/unknown etiology (vs. LAA)

$1.49(1.05-2.23)$
$0.78(0.54-1.07)$
$1.63(1.06-2.74)$
$0.78(0.46-1.18)$
$0.84(0.41-1.05)$
$1.10(0.85-2.28)$
$0.86(0.78-0.94)$
$3.14(1.03-10.7)$
$4.89(1.48-19.1)$
$0.81(0.19-3.23)$

0.024

$1.57(1.12-2.35)$

0.007

0.129

$0.73(0.47-1.05)$

0.098

0.023

$2.29(1.16-5.64)$

0.014

0.262

$0.59(0.23-1.17)$

0.145

0.321

$0.77(0.36-1.03)$

0.205

0.743

$1.18(0.84-2.54)$

0.610

$<0.001$

$0.87(0.79-0.94)$

$<0.001$

0.043

$2.74(0.88-9.43)$

0.082

0.008

4.81 (1.44-18.9)

0.009

0.763

$0.81(0.19-3.27)$

0.767

$0.23(0.06-0.82)$

0.022

$0.19(0.04-0.72)$

0.013

$5.73(1.58-25.1)$

0.007

$4.66(1.27-20.5)$

0.019

$0.30(0.10-0.79)$

0.014

$0.30(0.11-0.79)$

0.015

$0.74(0.51-1.04)$

$0.78(0.54-1.09)$

0.143

Model fit statistics (LL, AIC, BIC)

$-71.406,172.81,218.46$

$-70.405,170.81,216.46$

Full B: with excursions*reference interaction

Default independent variables

Rate of BP $>120 \%$ of reference (by $1 / 10 \mathrm{~min}$ )

$1.55(1.01-2.47)$

0.043

$1.59(1.03-2.64)$

0.034

$0.89(0.55-1.40)$

0.624

$0.74(0.44-1.15)$

0.192

$1.72(1.09-3.01)$

0.017

$2.30(1.13-5.84)$

0.019

$1.00(0.85-1.18)$

0.995

1.01 (0.84-1.24)

0.948

0.97 (0.89-1.05)

0.409

$1.00(0.89-1.13)$

0.969

$0.76(0.44-1.17)$

0.228

$0.59(0.23-1.18)$

0.150

$0.81(0.38-1.04)$

0.291

$0.77(0.36-1.04)$

0.216

$1.14(0.85-2.44)$

0.672

$1.18(0.84-2.55)$

0.616

$0.86(0.78-0.94)$

$<0.001$

$3.16(1.03-10.8)$

0.043

0.87 (0.79-0.94)

$<0.001$

$\mathrm{TICl}$ grade $2 \mathrm{~b}-3$ (vs. 0-2a)

Collaterals $\geq 50 \%$ as unaffected side (vs. no)

5.53 (1.61-23.0)

$2.73(0.88-9.48)$

0.084

0.006

4.84 (1.43-19.4)

0.011

$0.78(0.18-3.15)$

0.81 (0.19-3.29)

0.768

Selected independent variables ${ }^{\dagger}$

Cardioembolic/unknown etiology (vs. LAA)

Pre-index stroke statin use

$0.19(0.04-0.74)$

0.015

$5.70(1.59-24.4)$

0.007

4.67 (1.27-20.7)

0.019

$0.29(0.10-0.78)$

0.003

$0.30(0.11-0.80)$

0.015

$0.74(0.51-1.04)$

0.78 (0.54-1.09)

0.148

Age (by $10 \mathrm{yr}$ )

Model fit statistics (LL, AIC, BIC)

$-71.059,176.12,227.86$

$-70.402,174.80,226.54$

Full C: with excursions* $* \mathrm{ICl}$ interaction

$$
\begin{aligned}
& \text { Rate of } \mathrm{BP}>120 \% \text { of reference* } * \mathrm{TICl} \text { grade } \\
& \text { Rate of } \mathrm{BP}>120 \% \text { at } \mathrm{TICl} 0-2 \mathrm{a} \\
& \text { Rate of } \mathrm{BP}>120 \% \text { at } \mathrm{TICl} 2 \mathrm{~b}-3 \\
& \text { Rate of } \mathrm{BP}<80 \% \text { of reference } * \mathrm{TICl} \text { grade }
\end{aligned}
$$$$
7.72(1.12-53.3)
$$$$
0.038
$$$$
2.44(0.84-6.63)
$$$$
0.080
$$$$
0.27(0.04-1.75)
$$$$
-
$$$$
0.74(0.28-1.99)
$$$$
2.10(1.25-3.51)
$$

$1.15(0.70-1.89)$

$1.81(1.19-2.75)$

0.589

$1.06(0.57-1.96)$

0.865 
Supplementary Table 5. Continued

\begin{tabular}{|c|c|c|c|c|}
\hline \multirow{2}{*}{ Model } & \multicolumn{2}{|c|}{ Systolic blood pressure } & \multicolumn{2}{|c|}{ Mean arterial pressure } \\
\hline & $\mathrm{OR}(95 \% \mathrm{Cl})$ & $P$ & $\mathrm{OR}(95 \% \mathrm{Cl})$ & $P$ \\
\hline Rate of $\mathrm{BP}<80 \%$ at $\mathrm{TICl} 0-2 \mathrm{a}$ & $0.71(0.41-1.22)$ & - & $0.74(0.39-1.40)$ & - \\
\hline Rate of $\mathrm{BP}<80 \%$ at $\mathrm{TICl} 2 \mathrm{~b}-3$ & $0.82(0.55-1.20)$ & - & $0.78(0.51-1.17)$ & - \\
\hline Reference BP (by 10 mm Hg) & $1.62(1.02-2.79)$ & 0.038 & $2.12(1.08-5.12)$ & 0.028 \\
\hline Procedure mean BP (by $10 \mathrm{~mm} \mathrm{Hg})^{+}$ & $0.78(0.45-1.21)$ & 0.292 & $0.65(0.26-1.28)$ & 0.238 \\
\hline $\begin{array}{l}\text { TICl grade } 2 \mathrm{~b}-3 \text { (vs. } 0-2 \mathrm{a} \text { ) } \\
\text { (other effects were closely similar to } \mathrm{A} \text { and } \mathrm{B} \text { ) }\end{array}$ & $7.36(1.15-47.1)$ & 0.035 & $3.67(0.97-13.9)$ & 0.056 \\
\hline Model fit statistics (LL, AIC, BIC) & $-66.396,166.79,218.53$ & & $-67.909,169.82,221.56$ & \\
\hline \multicolumn{5}{|l|}{ Full D: with $\mathrm{BP} * \mathrm{TICl}$ interaction } \\
\hline Rate of $\mathrm{BP}>120 \%$ of reference (by $1 / 10 \mathrm{~min}$ ) & $1.56(1.03-2.38)$ & 0.037 & $1.62(1.08-2.41)$ & 0.019 \\
\hline Rate of $\mathrm{BP}<80 \%$ of reference (by $1 / 10 \mathrm{~min}$ ) & $0.77(0.53-1.11)$ & 0.182 & $0.74(0.49-1.12)$ & 0.153 \\
\hline $\mathrm{TICl}$ grade $2 \mathrm{~b}-3$ (vs. $0-2 \mathrm{a})$ & $3.60(1.09-11.8)$ & 0.035 & $3.17(0.93-10.8)$ & 0.065 \\
\hline Reference $\mathrm{BP} * \mathrm{TICl}$ grade & $0.71(0.42-1.19)$ & 0.191 & $0.51(0.21-1.21)$ & 0.125 \\
\hline Reference $\mathrm{BP}$ at $\mathrm{TICl} 0-2 \mathrm{a}$ & $2.16(1.09-4.29)$ & - & $3.97(1.29-12.3)$ & - \\
\hline Reference $\mathrm{BP}$ at $\mathrm{TICl} 2 \mathrm{~b}-3$ & $1.53(0.92-2.55)$ & - & $2.02(0.88-4.61)$ & - \\
\hline Procedure mean $\mathrm{BP} * \mathrm{TICl}$ grade & $1.45(0.71-1.99)$ & 0.310 & $2.76(0.85-8.98)$ & 0.093 \\
\hline Procedure mean $\mathrm{BP}$ at $\mathrm{TICl} 0-2 \mathrm{a}$ & $0.57(0.26-1.29)$ & - & $0.26(0.07-1.01)$ & - \\
\hline $\begin{array}{l}\text { Procedure mean } \mathrm{BP} \text { at } \mathrm{TICl} 2 \mathrm{~b}-3 \\
\text { (other effects were closely similar to } \mathrm{A} \text { and } \mathrm{B} \text { ) }\end{array}$ & $0.84(0.50-1.39)$ & - & $0.72(0.30-1.73)$ & - \\
\hline Model fit statistics (LL, AIC, BIC) & $-70.385,174.77,226.51$ & & $-68.755,171.51,223.25$ & \\
\hline \multicolumn{5}{|l|}{ Reduced A: no interactions, no CT findings ${ }^{\S}$} \\
\hline \multicolumn{5}{|l|}{ Default independent variables } \\
\hline Rate of $\mathrm{BP}>120 \%$ of reference (by $1 / 10 \mathrm{~min}$ ) & $1.44(1.04-2.06)$ & 0.026 & $1.38(1.03-1.83)$ & 0.023 \\
\hline Rate of $\mathrm{BP}<80 \%$ of reference (by $1 / 10 \mathrm{~min}$ ) & $0.78(0.57-1.05)$ & 0.101 & $0.84(0.64-1.10)$ & 0.193 \\
\hline Reference BP (by 10 mm Hg) & $1.58(1.07-2.48)$ & 0.022 & $1.75(1.00-3.07)$ & 0.042 \\
\hline Procedure mean BP (by $10 \mathrm{~mm} \mathrm{Hg})^{+}$ & $0.84(0.56-1.21)$ & 0.360 & $0.80(0.47-1.36)$ & 0.405 \\
\hline On-admission NIHSS score (by 1 point) & $0.86(0.78-0.93)$ & $<0.001$ & $0.86(0.79-0.94)$ & $<0.001$ \\
\hline $\mathrm{TICl}$ grade $2 \mathrm{~b}-3$ (vs. $0-2 a)$ & $4.12(1.44-13.3)$ & 0.008 & $3.85(1.29-11.5)$ & 0.011 \\
\hline Collaterals $\geq 50 \%$ as unaffected side (vs. no) & $4.81(1.53-17.6)$ & 0.007 & $4.81(1.43-16.1)$ & 0.007 \\
\hline \multicolumn{5}{|l|}{ Selected independent variables ${ }^{\ddagger}$} \\
\hline Cardioembolic/unknown etiology (vs. LAA) & $0.27(0.07-0.92)$ & 0.036 & $0.27(0.07-0.97)$ & 0.035 \\
\hline Pre-index stroke statin use & $5.77(1.64-24.2)$ & 0.005 & $4.55(1.24-16.7)$ & 0.015 \\
\hline Post-index stroke statin use & $0.29(0.11-0.75)$ & 0.010 & $0.31(0.12-0.81)$ & 0.013 \\
\hline Age (by 10 yr) & $0.69(0.49-0.94)$ & 0.020 & $0.72(0.52-0.99)$ & 0.037 \\
\hline Model fit statistics (LL, AIC, BIC) & $-73.084,170.17,206.69$ & & $-70.405,170.81,216.46$ & \\
\hline \multicolumn{5}{|l|}{ Reduced B: no interactions, with CT findings ${ }^{\S}$} \\
\hline \multicolumn{5}{|l|}{ Default independent variables } \\
\hline Rate of $\mathrm{BP}>120 \%$ of reference (by $1 / 10 \mathrm{~min}$ ) & $1.40(0.98-2.06)$ & 0.059 & $1.31(0.96-1.80)$ & 0.079 \\
\hline Rate of $\mathrm{BP}<80 \%$ of reference (by $1 / 10 \mathrm{~min}$ ) & $0.81(0.57-1.11)$ & 0.190 & $0.88(0.66-1.18)$ & 0.387 \\
\hline Reference BP (by 10 mm Hg) & $1.56(1.01-2.60)$ & 0.042 & $1.61(0.87-2.99)$ & 0.111 \\
\hline Procedure mean BP (by $10 \mathrm{~mm} \mathrm{Hg})^{+}$ & $0.83(0.52-1.24)$ & 0.363 & $0.80(0.44-1.44)$ & 0.443 \\
\hline On-admission NIHSS score (by 1 point) & $0.87(0.79-0.95)$ & 0.002 & $0.88(0.81-0.96)$ & 0.004 \\
\hline $\mathrm{TICl}$ grade $2 \mathrm{~b}-3$ (vs. $0-2 \mathrm{a})$ & $3.02(0.95-10.6)$ & 0.061 & $2.78(0.86-8.97)$ & 0.080 \\
\hline Collaterals $\geq 50 \%$ as unaffected side (vs. no) & $3.12(0.91-12.2)$ & 0.071 & $3.27(0.90-11.9)$ & 0.061 \\
\hline Ischemic lesion volume (by 2.718-fold) & $0.62(0.46-0.80)$ & $<0.001$ & $0.62(0.47-0.82)$ & $<0.001$ \\
\hline Post-procedure visible hemorrhages (vs. no) & $1.87(0.55-6.34)$ & 0.314 & $1.88(0.57-6.22)$ & 0.298 \\
\hline
\end{tabular}


Supplementary Table 5. Continued

\begin{tabular}{|c|c|c|c|c|}
\hline \multirow{2}{*}{ Model } & \multicolumn{2}{|c|}{ Systolic blood pressure } & \multicolumn{2}{|c|}{ Mean arterial pressure } \\
\hline & OR $(95 \% \mathrm{Cl})$ & $P$ & OR $(95 \% \mathrm{Cl})$ & $P$ \\
\hline \multicolumn{5}{|l|}{ Selected independent variables ${ }^{*}$} \\
\hline Cardioembolic/unknown etiology (vs. LAA) & $0.18(0.04-0.68)$ & 0.011 & $0.17(0.04-0.70)$ & 0.009 \\
\hline Pre-index stroke statin use & $3.62(0.97-15.7)$ & 0.055 & $3.19(0.82-12.5)$ & 0.082 \\
\hline Post-index stroke statin use & $0.31(0.11-0.82)$ & 0.018 & $0.31(0.11-0.84)$ & 0.017 \\
\hline Age (by 10 yr) & $0.62(0.42-0.87)$ & 0.005 & $0.72(0.52-0.99)$ & 0.008 \\
\hline Model fit statistics (LL, AIC, BIC) & $-66.213,160.42,203.03$ & & $-67.075,162.15,204.76$ & \\
\hline \multicolumn{5}{|l|}{ Reduced C: with interaction, no CT findings ${ }^{\S}$} \\
\hline \multicolumn{5}{|l|}{ Default independent variables } \\
\hline Rate of $\mathrm{BP}>120 \%$ of reference $* \mathrm{TICl}$ grade & $8.03(1.19-54.3)$ & 0.033 & $2.64(0.98-7.05)$ & 0.053 \\
\hline Rate of $\mathrm{BP}>120 \%$ at $\mathrm{TICI} 0-2 \mathrm{a}$ & $0.25(0.04-1.56)$ & - & $0.62(0.24-1.59)$ & - \\
\hline Rate of $\mathrm{BP}>120 \%$ at $\mathrm{TICl} 2 \mathrm{~b}-3$ & $1.98(1.24-3.17)$ & - & $1.64(1.15-2.33)$ & - \\
\hline Rate of $\mathrm{BP}<80 \%$ of reference (by $1 / 10 \mathrm{~min}$ ) & $0.81(0.59-1.11)$ & 0.182 & $0.80(0.57-1.12)$ & 0.202 \\
\hline Reference BP (by 10 mm Hg) & $1.54(1.00-2.38)$ & 0.048 & $1.81(1.01-3.62)$ & 0.046 \\
\hline Procedure mean BP (by $10 \mathrm{~mm} \mathrm{Hg}^{+}$ & $0.86(0.59-1.26)$ & 0.447 & $0.80(0.45-1.43)$ & 0.458 \\
\hline On-admission NIHSS score (by 1 point) & $0.84(0.76-0.92)$ & $<0.001$ & $0.86(0.79-0.94)$ & $<0.001$ \\
\hline $\mathrm{TICl}$ grade $2 \mathrm{~b}-3$ (vs. $0-2 \mathrm{a})$ & $11.1(1.84-66.2)$ & 0.008 & $5.30(1.49-18.8)$ & 0.010 \\
\hline Collaterals $\geq 50 \%$ as unaffected side (vs. no) & $5.07(1.47-17.5)$ & 0.010 & $5.78(1.68-19.9)$ & 0.005 \\
\hline \multicolumn{5}{|l|}{ Selected independent variables ${ }^{\dagger}$} \\
\hline Cardioembolic/unknown etiology (vs. LAA) & $0.28(0.07-1.12)$ & 0.071 & $0.27(0.07-1.07)$ & 0.062 \\
\hline Pre-index stroke statin use & $6.26(1.52-25.7)$ & 0.011 & $4.48(1.18-17.1)$ & 0.028 \\
\hline Post-index stroke statin use & $0.25(0.09-0.69)$ & 0.007 & $0.28(0.10-0.74)$ & 0.011 \\
\hline Age (by 10 yr) & $0.66(0.47-0.93)$ & 0.018 & $0.67(0.45-0.95)$ & 0.025 \\
\hline Model fit statistics (LL, AIC, BIC) & $-68.076,162.15,201.72$ & & $-70.079,166.16,205.72$ & \\
\hline \multicolumn{5}{|l|}{ Reduced D: with interaction and CT findings ${ }^{\S}$} \\
\hline \multicolumn{5}{|l|}{ Default independent variables } \\
\hline Rate of $\mathrm{BP}>120 \%$ of reference $* \mathrm{TICl}$ grade $^{*}$ & $7.55(0.91-62.3)$ & 0.061 & $2.51(0.92-6.81)$ & 0.071 \\
\hline Rate of $\mathrm{BP}>120 \%$ at $\mathrm{TICl} 0-2 \mathrm{a}$ & $0.24(0.03-1.89)$ & - & $0.61(0.23-1.59)$ & - \\
\hline Rate of $\mathrm{BP}>120 \%$ at $\mathrm{TICl} 2 b-3$ & $1.84(1.13-3.00)$ & - & $1.53(1.05-2.21)$ & - \\
\hline Rate of BP $<80 \%$ of reference (by $1 / 10 \mathrm{~min}$ ) & $0.83(0.59-1.16)$ & 0.272 & $0.83(0.57-1.19)$ & 0.312 \\
\hline Reference BP (by 10 mm Hg) & $1.52(0.90-2.53)$ & 0.056 & $1.71(0.90-3.73)$ & 0.103 \\
\hline Procedure mean BP (by $10 \mathrm{~mm} \mathrm{Hg})^{+}$ & $0.86(0.56-1.30)$ & 0.471 & $0.79(0.41-1.52)$ & 0.485 \\
\hline On-admission NIHSS score (by 1 point) & $0.86(0.78-0.96)$ & 0.005 & $0.88(0.80-0.97)$ & 0.007 \\
\hline $\mathrm{TICl}$ grade $2 \mathrm{~b}-3$ (vs. $0-2 \mathrm{a}$ ) & $8.21(1.07-62.8)$ & 0.042 & $3.70(0.95-14.4)$ & 0.059 \\
\hline Collaterals $\geq 50 \%$ as unaffected side (vs. no) & $3.33(0.90-12.3)$ & 0.072 & $3.88(1.05-14.3)$ & 0.042 \\
\hline Ischemic lesion volume (by 2.718-fold) & $0.63(0.48-0.83)$ & 0.001 & $0.63(0.48-0.84)$ & 0.001 \\
\hline Post-procedure visible hemorrhages (vs. no) & $1.75(0.50-6.15)$ & 0.380 & $1.63(0.48-5.54)$ & 0.435 \\
\hline \multicolumn{5}{|l|}{ Selected independent variables ${ }^{*}$} \\
\hline Cardioembolic/unknown etiology (vs. LAA) & $0.19(0.04-0.91)$ & 0.036 & $0.17(0.04-0.77)$ & 0.021 \\
\hline Pre-index stroke statin use & $3.91(0.91-16.8)$ & 0.066 & $3.18(0.79-12.9)$ & 0.104 \\
\hline Post-index stroke statin use & $0.25(0.09-0.73)$ & 0.011 & $0.28(0.10-0.77)$ & 0.013 \\
\hline Age (by $10 \mathrm{yr}$ ) & $0.60(0.41-0.86)$ & 0.006 & $0.60(0.41-0.98)$ & 0.009 \\
\hline Model fit statistics (LL, AIC, BIC) & $-62.144,154.29,199.94$ & & $-64.446,158.89,204.54$ & \\
\hline \multicolumn{5}{|l|}{ Final A: no interactions, no CT findings } \\
\hline \multicolumn{5}{|l|}{ Default independent variables } \\
\hline Rate of $\mathrm{BP}>120 \%$ of reference (by $1 / 10 \mathrm{~min}$ ) & $1.33(1.01-1.76)$ & 0.038 & $1.30(1.01-1.66)$ & 0.029 \\
\hline
\end{tabular}


Supplementary Table 5. Continued

\begin{tabular}{|c|c|c|c|c|}
\hline \multirow{2}{*}{ Model } & \multicolumn{2}{|c|}{ Systolic blood pressure } & \multicolumn{2}{|c|}{ Mean arterial pressure } \\
\hline & OR $(95 \% \mathrm{Cl})$ & $P$ & OR $(95 \% \mathrm{Cl})$ & $P$ \\
\hline Rate of $\mathrm{BP}<80 \%$ of reference (by $1 / 10 \mathrm{~min}$ ) & $0.85(0.67-1.08)$ & 0.174 & $0.85(0.65-1.10)$ & 0.218 \\
\hline Reference BP (by $10 \mathrm{~mm} \mathrm{Hg}$ ) & $1.37(1.06-1.77)$ & 0.013 & $1.49(1.06-2.08)$ & 0.016 \\
\hline On-admission NIHSS score (by 1 point) & $0.85(0.78-0.93)$ & $<0.001$ & $0.86(0.80-0.94)$ & $<0.001$ \\
\hline $\mathrm{TICl}$ grade $2 b-3$ (vs. $0-2 a)$ & $3.96(1.34-11.7)$ & 0.009 & $3.72(1.26-11.0)$ & 0.013 \\
\hline Collaterals $\geq 50 \%$ as unaffected side (vs. no) & $4.62(1.45-11.2)$ & 0.007 & $4.71(1.44-15.4)$ & 0.006 \\
\hline \multicolumn{5}{|l|}{ Selected independent variables ${ }^{\dagger}$} \\
\hline Cardioembolic/unknown etiology (vs. LAA) & $0.25(0.07-090)$ & 0.026 & $0.22(0.06-0.84)$ & 0.018 \\
\hline Pre-index stroke statin use & $5.88(1.55-22.3)$ & 0.005 & $4.71(1.27-17.5)$ & 0.013 \\
\hline Post-index stroke statin use & $0.32(0.12-0.82)$ & 0.015 & $0.34(0.13-0.87)$ & 0.020 \\
\hline Age (by 10 yr) & $0.69(0.50-0.96)$ & 0.020 & $0.72(0.52-0.99)$ & 0.039 \\
\hline Model fit statistics (LL, AIC, BIC) & $-73.504,169.01,202.48$ & & $-73.544,169.09,202.56$ & \\
\hline \multicolumn{5}{|l|}{ Final B: no interactions, with lesion volume } \\
\hline \multicolumn{5}{|l|}{ Default independent variables } \\
\hline Rate of $B P>120 \%$ of reference (by $1 / 10 \mathrm{~min}$ ) & $1.25(0.95-1.71)$ & 0.114 & $1.22(0.95-1.61)$ & 0.115 \\
\hline Rate of $\mathrm{BP}<80 \%$ of reference (by $1 / 10 \mathrm{~min}$ ) & $0.88(0.69-1.11)$ & 0.291 & $0.89(0.67-1.15)$ & 0.371 \\
\hline Reference BP (by $10 \mathrm{~mm} \mathrm{Hg}$ ) & $1.33(1.02-1.77)$ & 0.034 & $1.37(0.97-1.97)$ & 0.071 \\
\hline On-admission NIHSS score (by 1 point) & $0.87(0.79-0.95)$ & 0.002 & $0.88(0.81-0.96)$ & 0.003 \\
\hline $\mathrm{TICl}$ grade $2 \mathrm{~b}-3$ (vs. $0-2 \mathrm{a})$ & $2.73(0.89-9.10)$ & 0.079 & $2.55(0.83-8.46)$ & 0.101 \\
\hline Collaterals $\geq 50 \%$ as unaffected side (vs. no) & $2.95(0.88-11.2)$ & 0.080 & $3.04(0.91-11.5)$ & 0.071 \\
\hline Ischemic lesion volume (by 2.718-fold) & $0.65(0.50-0.83)$ & $<0.001$ & $0.66(0.50-0.84)$ & $<0.001$ \\
\hline \multicolumn{5}{|l|}{ Selected independent variables ${ }^{*}$} \\
\hline Cardioembolic/unknown etiology (vs. LAA) & $0.19(0.05-0.69)$ & 0.011 & $0.16(0.04-0.62)$ & 0.007 \\
\hline Pre-index stroke statin use & $3.94(1.08-16.8)$ & 0.037 & $3.48(0.97-14.8)$ & 0.057 \\
\hline Post-index stroke statin use & $0.34(0.12-0.88)$ & 0.026 & $0.33(0.12-0.87)$ & 0.025 \\
\hline Age (by 10 yr) & $0.63(0.43-0.87)$ & 0.005 & $0.65(0.45-0.90)$ & 0.010 \\
\hline Model fit statistics (LL, AIC, BIC) & $-67.354,158.71,195.23$ & & $-67.863,159.73,196.25$ & \\
\hline
\end{tabular}

Final $\mathrm{C}$ : with interaction, no CT findings

Default independent variables

Rate of $\mathrm{BP}>120 \%$ of reference $* \mathrm{TICl}$ grade

Rate of $\mathrm{BP}>120 \%$ at $\mathrm{TICl} 0-2 \mathrm{a}$

Rate of $\mathrm{BP}>120 \%$ at $\mathrm{TICl} 2 \mathrm{~b}-3$

$\mathrm{TICl}$ grade $2 b-3$ (vs. $0-2 \mathrm{a}$ ) at 0 rate

$\mathrm{TICl}$ grade $2 \mathrm{~b}-3$ (vs. 0-2a) at 0.5/10 $\mathrm{min}$

$\mathrm{TICl}$ grade $2 \mathrm{~b}-3$ (vs. $0-2 \mathrm{a}$ ) at $1.0 / \mathrm{min}$

$\mathrm{TICl}$ grade $2 \mathrm{~b}-3$ (vs. 0-2a) at mean rate

Rate of $\mathrm{BP}<80 \%$ of reference (by $1 / 10 \mathrm{~min}$ )

Reference BP (by $10 \mathrm{~mm} \mathrm{Hg}$ )

On-admission NIHSS score (by 1 point)

Collaterals $\geq 50 \%$ as unaffected side (vs. no)

Selected independent variables ${ }^{\dagger}$

Cardioembolic/unknown etiology (vs. LAA)

Pre-index stroke statin use

Post-index stroke statin use

Age (by $10 \mathrm{yr}$ )

Model fit statistics (LL, AIC, BIC)

\begin{tabular}{lllc}
$8.42(1.25-56.9)$ & 0.029 & $2.80(1.05-7.43)$ & 0.039 \\
$0.22(0.04-1.35)$ & - & $0.55(0.23-1.36)$ & - \\
$1.84(1.20-2.82)$ & - & $1.55(1.12-2.14)$ & - \\
$1.01(0.25-3.97)$ & - & $1.33(0.35-5.10)$ & - \\
$2.92(0.92-9.27)$ & - & $2.23(0.71-7.03)$ & - \\
$8.48(1.69-42.6)$ & - & $3.73(1.19-11.7)$ & - \\
$11.1(1.84-66.2)$ & 0.009 & $5.37(1.52-18.9)$ & 0.009 \\
$0.87(0.69-1.11)$ & 0.257 & $0.87(0.67-1.12)$ & 0.285 \\
$1.36(1.04-1.78)$ & 0.026 & $1.50(1.06-2.12)$ & 0.022 \\
$0.84(0.76-0.92)$ & $<0.001$ & $0.86(0.78-0.93)$ & $<0.001$ \\
$5.07(1.47-17.5)$ & 0.010 & $5.68(1.67-19.4)$ & 0.009 \\
$0.28(0.07-1.12)$ & & & \\
$6.26(1.52-25.7)$ & 0.072 & $0.26(0.06-1.03)$ & 0.054 \\
$0.25(0.09-0.69)$ & 0.011 & $4.66(1.22-17.8)$ & 0.025 \\
$0.66(0.47-0.93)$ & 0.007 & $0.29(0.11-0.78)$ & 0.014 \\
$68.374,160.75,197.27$ & 0.017 & $0.67(0.48-0.95)$ & 0.025 \\
& & $-70.370,164.74,201.26$ & \\
& & & \\
\hline
\end{tabular}


Supplementary Table 5. Continued

\begin{tabular}{|c|c|c|c|c|}
\hline \multirow{2}{*}{ Model } & \multicolumn{2}{|c|}{ Systolic blood pressure } & \multicolumn{2}{|c|}{ Mean arterial pressure } \\
\hline & OR $(95 \% \mathrm{Cl})$ & $P$ & OR (95\% Cl) & $P$ \\
\hline \multicolumn{5}{|l|}{ Final D: with interaction, with lesion volume } \\
\hline \multicolumn{5}{|l|}{ Default independent variables } \\
\hline Rate of $\mathrm{BP}>120 \%$ of reference $* \mathrm{TICl}$ grade & $7.86(0.99-62.1)$ & 0.051 & $2.73(1.02-7.31)$ & 0.046 \\
\hline Rate of $\mathrm{BP}>120 \%$ at $\mathrm{TICl} 0-2 \mathrm{a}$ & $0.21(0.03-1.55)$ & - & $0.53(0.21-1.31)$ & - \\
\hline Rate of $\mathrm{BP}>120 \%$ at $\mathrm{TICl} 2 \mathrm{~b}-3$ & $1.67(1.08-2.60)$ & - & $1.43(1.03-2.01)$ & - \\
\hline $\mathrm{TICl}$ grade $2 b-3$ (vs. $0-2 a$ ) at 0 rate & $0.77(0.18-3.20)$ & - & $0.94(0.23-3.83)$ & - \\
\hline $\mathrm{TICl}$ grade $2 \mathrm{~b}-3$ (vs. $0-2 \mathrm{a}$ ) at $0.5 / 10 \mathrm{~min}$ & $2.16(0.62-7.56)$ & - & $1.55(0.46-5.24)$ & - \\
\hline $\mathrm{TICl}$ grade $2 \mathrm{~b}-3$ (vs. $0-2 \mathrm{a}$ ) at $1.0 / \mathrm{min}$ & $6.05(0.99-36.6)$ & - & $2.56(0.76-8.64)$ & - \\
\hline $\mathrm{TICl}$ grade $2 \mathrm{~b}-3$ (vs. $0-2 a$ ) at mean rate & $7.21(1.29-59.1)$ & 0.021 & $3.64(0.96-13.8)$ & 0.057 \\
\hline Rate of $\mathrm{BP}<80 \%$ of reference (by $1 / 10 \mathrm{~min}$ ) & $0.89(0.69-1.13)$ & 0.341 & $0.89(0.68-1.15)$ & 0.389 \\
\hline Reference BP (by $10 \mathrm{~mm} \mathrm{Hg}$ ) & $1.33(1.01-1.79)$ & 0.040 & $1.39(0.98-2.02)$ & 0.067 \\
\hline On-admission NIHSS score (by 1 point) & $0.86(0.77-0.95)$ & 0.002 & $0.88(0.80-0.96)$ & 0.004 \\
\hline Collaterals $\geq 50 \%$ as unaffected side (vs. no) & $3.35(0.97-13.2)$ & 0.056 & $3.81(1.11-14.9)$ & 0.033 \\
\hline Ischemic lesion volume (by 2.718-fold) & $0.66(0.51-0.85)$ & 0.001 & $0.66(0.51-0.85)$ & 0.001 \\
\hline \multicolumn{5}{|l|}{ Selected independent variables ${ }^{*}$} \\
\hline Cardioembolic/unknown etiology (vs. LAA) & $0.22(0.05-0.85)$ & 0.028 & $0.19(0.04-0.85)$ & 0.015 \\
\hline Pre-index stroke statin use & $4.28(1.08-19.6)$ & 0.038 & $3.44(0.92-15.1)$ & 0.068 \\
\hline Post-index stroke statin use & $0.27(0.09-0.74)$ & 0.010 & $0.29(0.10-0.78)$ & 0.013 \\
\hline Age (by 10 yr) & $0.61(0.41-0.86)$ & 0.004 & $0.61(0.41-0.87)$ & 0.005 \\
\hline Model fit statistics (LL, AIC, BIC) & $-62.945,151.89,191.45$ & & $-65.136,156.27,195.83$ & \\
\hline
\end{tabular}

Three types of models (each separately for systolic and mean arterial pressure) were fitted: "full" (with different interaction terms), "reduced" and "final" (based on biological and statistical plausibility). Intermediate radiological outcomes were introduced to "reduced models". Effects are ORs. ORs for the interaction terms indicate whether the BP excursion effects were conditional on reference BP; or whether BP excursion effects and the effects of reference and procedure mean BP were conditional on the level of reperfusion (TICl grade).

$\mathrm{BP}$, blood pressure; EVT, endovascular thrombectomy; mRS, modified Rankin Scale; OR, odds ratio; $\mathrm{Cl}$, confidence interval; NIHSS, National Institutes of Health Stroke Scale; TICI, Thrombolysis in Cerebral Infarction; LAA, large artery atherosclerosis; LL, logarithmic likelihood; AIC, Akaike information criterion; BIC, Bayesian information criterion; $\mathrm{CT}$, computed tomography.

${ }^{*}$ The asterisk is a part of the interaction term; ${ }^{+}$Weighted mean as area under the blood pressure-time curve divided by the time period covered; ${ }^{*}$ By stepwise selection procedure $(P<0.200$ to enter/stay) among: age, sex, comorbidities (hypertension, atrial fibrillation, any form of occlusive arterial disease, diabetes, heart or renal failure), stroke etiology, type of the affected vessel, use of recombinant tissue plasminogen activator, time elapsed since symptom onset till first

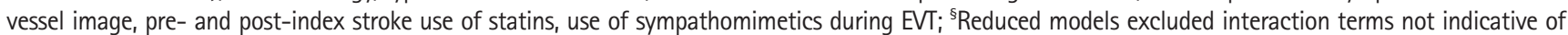
qualitative moderation, use of antihypertensives before EVT (small number of treated patients \& highly insignificant), procedure duration and number of BP measurements taken (consistently highly insignificant). Final models further excluded procedure mean BP (highly insignificant, determined by excursion rates, see Supplementary Analysis of Blood Pressure) and presence of post-procedure hemorrhages (consistently highly insignificant). 

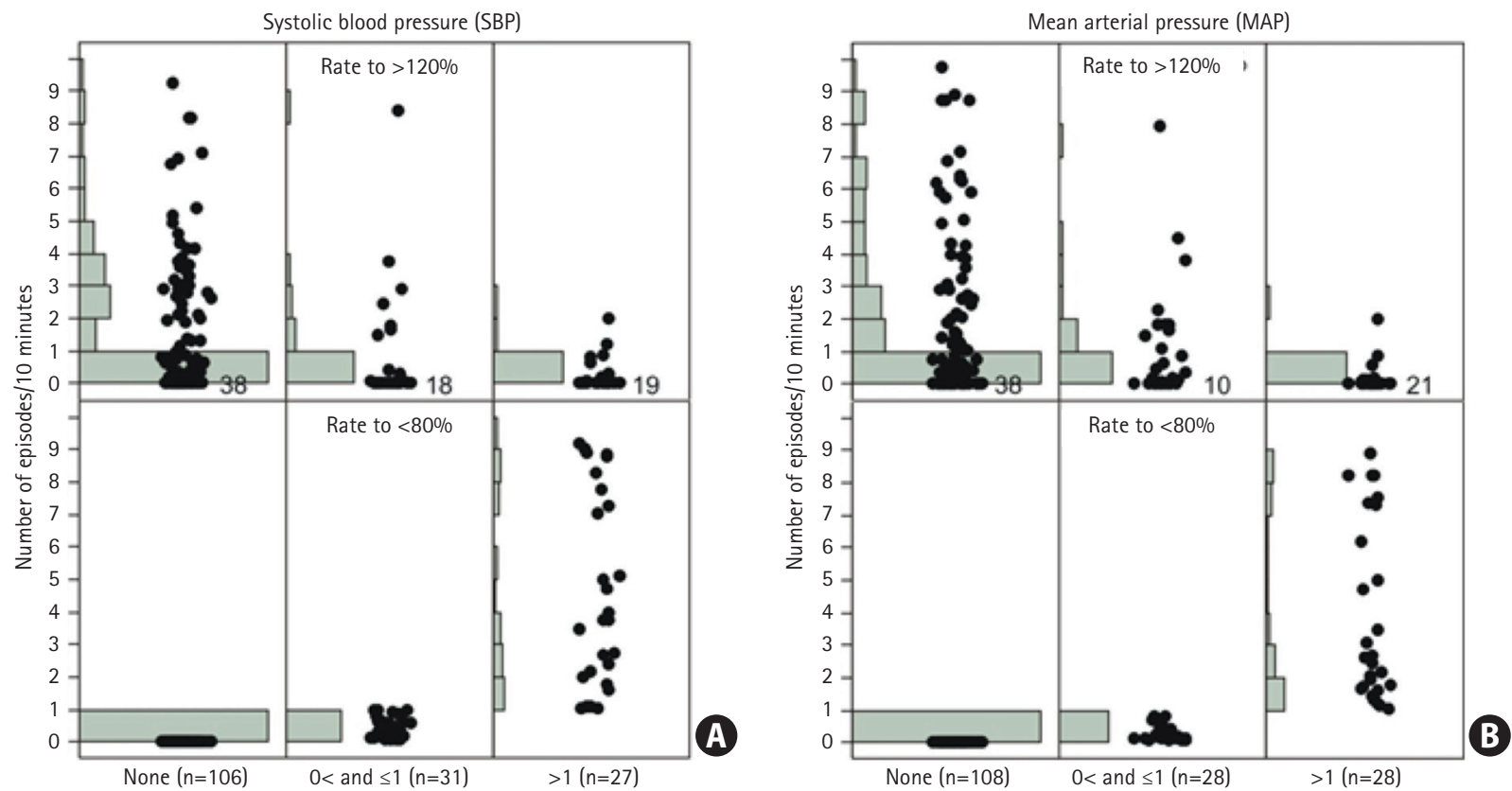

Patient categories by the rate of excursions to $>120 \%$ and $<80 \%$ of the reference $(\mathrm{n} / 10 \mathrm{~min}$ )

Supplementary Figure 1. Rates of blood pressure excursions during endovascular procedure to $>120 \%$ or to $<80 \%$ of the reference. Patients are classified by the rate of episodes ( $n / 10$ minutes) to $<80 \%$ as those with none, up to 1 and $>1$ episode/ 10 minutes, while actual rates are given on the $y$-axis: rates to $>120 \%$ of the reference (upper panel) and to $<80 \%$ of the reference (lower panel). Dots are individual data, shaded bars illustrate frequency of rates and numbers are counts of patients with no excursions to $>120 \%$ of the reference. 


\section{Supplementary Methods}

\section{Data analysis: multivariate model building procedures}

Building of all multivariate models was guided by the following general rationale: (a) indicators of in-procedure blood pressure (BP)/BP excursions from the reference value are independent variables of interest. All other potential independent variables serve to improve the control of confounding; (b) the sample size is rather limited, so models can sustain a limited number of independent variables; (c) selection of adjustments needs to follow (i) the nature of the independent variables of interest-they might be affected by procedure duration (i.e., have a "time component"), as well as by the number of measurements taken. BP excursions are defined in respect to reference values; hence, may be influenced by them; (ii) (patho)physiological rationale-certain factors, e.g., stroke severity at presentation (National Institutes of Health Stroke Scale [NIHSS] score), affected vessel(s), existence of leptomeningeal collaterals, use of pharmacological fibrinolysis (recombinant tissue plasminogen activator [rtPA]), the level of achieved reperfusion (Thrombolysis in Cerebral Infarction [ $\mathrm{TICl}$ grade $[\mathrm{TICl} 2 \mathrm{~b}-3=\mathrm{success} ; \mathrm{TICl}$ $0-2 a=f a i l u r e]$ ) may or are known to reflect on the control computed tomography findings (ischemic lesion volume [ILV], visible hemorrhages) and/or the 3-month functional outcome (modified Rankin Scale [mRS] score 0-2=favorable vs. 3-6=poor); hence, should be accounted for in the analysis of the respective outcomes; (iii) statistical significance-within the sample, some patient characteristics might be a source of confounding although without any obvious biological rationale and should be accounted for simply based on statistical significance; (d) consequently, all models need to include some adjustments by default, while others may be selected based on statistical significance; (e) some interactions need to be evaluated: is the potential effect of BP excursions from the reference value conditional on the reference value?; is it conditional on the level of reperfusion (TICl grade)?; is the potential effect of the reference or in-procedure BP conditional on the level of reperfusion?; ( $f$ ) there is a risk of overfitting; hence, models should be selected based on biological and statistical plausibility.

The logic and algorithm of model selection is depicted in Figure A. For all outcomes, i.e., imaging (intermediate) (ILV, post-procedure hemorrhages) and the primary clinical endpoint (mRS score 0-2), the procedure started by fitting "full" models including a range of default and selected variables. The initial model contained only main effects and further models sequentially tested interactions of interest. In the next step, models were "reduced" to keep independent variables of interest and biologically and statistically plausible covariates required to demonstrate hypothesized independent associations or lack of such associations. Imaging outcomes were considered as independent variables in the analysis of the 3-month mRS, but were not included in the full models; they were introduced to "reduced" models. Hence, model selection in the analysis of the primary clinical endpoint included a further step of selecting the "final" models.

All full models included the following same "base" default independent variables: rate of excursions to $>120 \%$ and to $<80 \%$ of the reference BP, reference $\mathrm{BP}$, weighted mean in-procedure BP, procedure duration (may also be a "proxy" of a more severe stroke/larger occlusion), number of in-procedure BP measurements, reperfusion success (TICl grade $2 b-3$ or $0-2 a$ ), existence of leptomeningeal collaterals $\geq 50 \%$ as on the unaffected side, on-admission NIHSS score and whether antihypertensive treatment was administered between admission and endovascular thrombectomy (EVT). Since data on the initial infarct volume were not available, we considered that on-admission NIHSS could be reasonably considered an independent that largely included (subsumed) the impact of the initial volume. We included also the use of antihypertensives between admission and EVT based on the following reasoning: decision to administer antihypertensives and subsequent (during EVT) decision to keep BP at lower levels/prevent excursions to higher values might have been guided by a larger (initial) infarct volume in order to prevent hemorrhagic transformation, and hence a spurious association between "better outcomes" (ILV, hemorrhages, 3-month functional outcome) and higher in-procedure BP, or "poorer outcomes" and lower in-procedure BP could be inferred. Therefore, on-admission NIHSS and use of antihypertensives between admission and EVT served as a kind of "proxy" to subsume the initial volume effects. A separate detailed analysis of on-admission BP, use of antihypertensives before EVT, use of sympathomimetics during EVT, weighted mean in-procedure BP and BP excursions was also performed (Supplementary Analysis of Blood Pressure).

In the analysis of the ILV, default adjustments additionally included the type of the affected vessel (middle cerebral artery segment 1 or segment 2, or involvement of internal carotid artery, i.e., tandem occlusion), while in the analysis of the presence of visible hemorrhages this was replaced with the use of rtPA as it seemed more plausible to account for a known risk factor for intracerebral hemorrhage. Only "base" default independent variables were included in the full models analyzing the 3-month functional outcome (probability of mRS 0-2 [vs. 3-6]).

In all full models, considered for inclusion through a stepwise selection procedure $(P<0.200$ to enter/stay) were: age, sex, comorbidities (hypertension, atrial fibrillation, any form of occlusive arterial disease, diabetes, heart or renal failure), stroke etiology by Trial of Org 10172 in Acute Stroke Treatment (TOAST) classification (dichotomized as cardioembolic or unknown vs. large artery atherosclerosis), time elapsed since symptom onset till the first vessel image, type of the affected vessel (if not by default), use of rtPA (if not by default), use of sympathomimetics during EVT and pre-index stroke statin use. In the analysis of ILV and visible hemorrhages considered were also pre-index stroke use of antiplatelets and anticoagulants, while in the analysis of the 3-month functional outcome post-procedure use of statins was considered instead.

Analysis of the 3-month functional outcome indicated a statistically significant interaction between the rate of BP excursions to $>120 \%$ of the reference and $\mathrm{TICl}$ grade achieved (Figure A), suggesting no association between excursions and the outcome in patients with $\mathrm{TICl}$ grade $0-2 \mathrm{a}$ and a strong association of a higher rate with higher odds of mRS 0-2 in patients with $\mathrm{TICl}$ grade 2b-3 (Supplementary Table 5). Conversely, it indicated no association between TICl $2 b-3$ and mRS 0-2 in patients with no BP excursions to $>120 \%$ of the reference, and increasingly stronger association with higher odds of mRS 0-2 with the increasing rate of excursions (Supplementary Table 5, Model final C, D). However, since there were only 40 patients with $\mathrm{TICl} 0-2 \mathrm{a}$, only 10 of whom achieved mRS 0-2, there is some uncertainty about this interaction. Therefore, two types of "final" models were generated (Figure A): not accounting and accounting for this interaction. We consider that models without the interaction generally better describe the data due to the mentioned uncertainty and: (a) lack of indication that systolic blood pressure (SBP)/mean arterial pressure (MAP) excursions in the subset of patients with TICl 0-2a were harmful-similar proportions of those with mRS 0-2 (4/10, 40\% for SBP, 20\% for MAP) and those with mRS 3-6 (20/30, 33.3\% for SBP, 20\% for MAP) had zero BP excursion rates to >120\% of the reference; (b) higher reference SBP/MAP was consistently associated with higher odds of mRS 0-2, both in patients with TICI 0-2a and with TICl $2 b-3$ (Supplementary Table 5); (c) at the start of EVT, TICl outcome is unknown. Present data indicate a potential benefit of higher rates of SBP/MAP excursions to $>120 \%$ and, at worst, no benefit (but no harm); (d) differences in formal statistical indicators of model fits (Bayesian information criterion [BIC]) between the final models without and with the interaction term were minor (Supplementary Table 5, Models final A and B vs. C and D). Finally, each of these models was fitted without and with an account for ILV (Figure A). Namely, higher ILV was consistently associated with lower odds of mRS 0-2 in the "reduced" and "final" models (Supplementary Table 5), but introduction of ILV had another consequence: strength of association between mRS 0-2 and several independent variables (BP excursion rates to $>120 \%$, existence of good collaterals, $\mathrm{TICl}$ grade, pre-index stroke statin use) was considerably reduced or the association was no more apparent (Supplementary Table 5). This phenomenon is typical for "mediator" variables and a possibility of mediated (via ILV) associations between BP excursion rates, existence of collaterals and TICl grade and 3-month mRS 0-2 appeared plausible: all these variables were also independently associated with 
ILV (Supplementary Table 3). Mediation analysis was performed specifically to test the hypothesis of an indirect association between in-procedure BP excursions to $>120 \%$ of the reference and the 3-month functional outcome via ILV (BP excursions $\rightarrow$ ILV $\rightarrow 3$-month mRS) with adjustments included in the "reduced" model analyzing ILV and "final" (without interactions) model analyzing mRS 0-2.

\begin{tabular}{|c|c|c|c|}
\hline \multicolumn{3}{|c|}{ Intermediate (computed tomography [CT]) outcomes } & Primary clinical endpoint \\
\hline Types of models & Ischemic lesion volume (ILV) & Post-procedure visible hemorrhages & mRS at 3 months 0-2 (vs. 3-6) \\
\hline $\begin{array}{l}\text { FULL } \\
\text { Initial: default and selected independents, } \\
\text { main effects, no interactions } \\
\text { Sequentially testing interactions: } \\
\text { a) rate of BP excursions*reference BP; } \\
\text { b) rate of excursions*ICl grade; } \\
\text { c) reference } B P * T I C I \text {, in-procedure mean } \\
\text { BP*IICI }\end{array}$ & $\begin{array}{l}\text { - Default: rate of BP excursions to }>120 \% \\
\text { of the reference, rate of excursions to }<80 \% \text {, } \\
\text { reference BP, in-procedure mean BP, EVT } \\
\text { duration, number of BP measurements taken, } \\
\text { on-admission NIHSS score, } \mathrm{TICl} \text { grade, } \\
\text { affected vessel, collaterals } \geq 50 \% \text { as } \\
\text { unaffected side, use of antihypertensives } \\
\text { before EVT } \\
\text { - Selected: sex }\end{array}$ & $\begin{array}{l}\text { - Default: rate of BP excursions to }>120 \% \\
\text { of the reference, rate of excursions to }<80 \% \text {, } \\
\text { reference BP, in-procedure mean BP, EVT } \\
\text { duration, number of BP measurements taken, } \\
\text { on-admission NIHSS score, TICl grade, use of } \\
\text { antihypertensives before EVT } \\
\text { - Selected: stroke etiology, history of } \\
\text { coronary artery disease }\end{array}$ & $\begin{array}{l}\text { - Default: rate of BP excursions to }>120 \% \\
\text { of the reference, rate of excursions to }<80 \% \text {, } \\
\text { reference BP, in-procedure mean BP, EVT } \\
\text { duration, number of BP measurements taken, } \\
\text { on-admission NIHSS score, } \mathrm{TICl} \text { grade, } \\
\text { collaterals } \geq 50 \% \text { as unaffected side, use of } \\
\text { antihypertensives before EVT } \\
\text { - Selected: stroke etiology, pre-/post-stroke } \\
\text { statin use, age }\end{array}$ \\
\hline $\begin{array}{l}\text { REDUCED } \\
\text { Keep: a) excursion rates (primary interest); } \\
\text { b) biologically \& statistically plausible } \\
\text { other effects (default or selected, } \\
\text { interactions) }\end{array}$ & $\begin{array}{l}\text { Kept effects: rate of BP excursions to } \\
>120 \% \text {, rate of BP excursions to }<80 \% \text {, } \\
\text { reference BP, rate to }>120 \% * \text { reference BP } \\
\text { interaction, NIHSS score, TICl grade, } \\
\text { collaterals, sex }\end{array}$ & $\begin{array}{l}\text { Kept effects: rate of BP excursions to } \\
>120 \% \text {, rate of BP excursions to }<80 \% \text {, } \\
\text { reference BP, in-procedure mean BP, NIHSS } \\
\text { score, TICI grade, stroke etiology, history of } \\
\text { coronary artery disease }\end{array}$ & $\begin{array}{l}\text { A. Without interaction and CT outcomes } \\
\text { Kept effects: rate of BP excursions to }>120 \% \\
\text { rate of } \mathrm{BP} \text { excursions to }<80 \% \text {, reference } \mathrm{BP} \text {, } \\
\text { in-procedure mean } \mathrm{BP}, \mathrm{NIHSS} \text { score, TICl } \\
\text { grade, collaterals, stroke etiology, }\end{array}$ \\
\hline \multirow{6}{*}{$\begin{array}{l}\text { FINAL } \\
\text { Further model reduction by final exclusion } \\
\text { of the initial default effects and } \\
\text { apparently irrelevant CT findings }\end{array}$} & & & $\begin{array}{l}\text { B. Without interaction, with CT outcomes } \\
\text { As A+ILV and hemorrhages }\end{array}$ \\
\hline & & & $\begin{array}{l}\text { C. With interaction, without CT outcomes } \\
\text { As A+rate to }>120 \% * * \mathrm{ICl} \text { interaction } \\
\text { D. With interaction and CT outcomes } \\
\text { As C+ILV and hemorrhages }\end{array}$ \\
\hline & & & $\begin{array}{l}\text { A. Without interaction and CT outcomes } \\
\text { Kept effects: rate of BP excursions to }>120 \% \\
\text { rate of BP excursions to }<80 \% \text {, reference } \mathrm{BP} \text {, } \\
\text { NIHSS score, TICl grade, collaterals, stroke } \\
\text { etiology, pre-/post-stroke statin use, age }\end{array}$ \\
\hline & & & $\begin{array}{l}\text { B. Without interaction and CT outcomes } \\
\text { As A+ILV }\end{array}$ \\
\hline & & & $\begin{array}{l}\text { C. With interaction, Without CT outcomes } \\
\text { As A+rate to }>120 \% * \mathrm{FICl} \text { interaction }\end{array}$ \\
\hline & & & $\begin{array}{l}\text { D. With interaction and CT outcomes } \\
\text { As C+ILV }\end{array}$ \\
\hline
\end{tabular}

Figure A. Schematic representation of the model selection strategy. All models were fitted separately for systolic blood pressure and mean arterial pressure. mRS, modified Rankin Scale; BP, blood pressure; TICl, Thrombolysis in Cerebral Infarction; EVT, endovascular thrombectomy; NIHSS, National Institutes of Health Stroke Scale; rtPA, recombinant tissue plasminogen activator. 


\title{
Supplementary Analysis of Blood Pressure
}

\section{Relationship between on-admission blood pressure (BP), BP at the start of endovascular thrombectomy (EVT), use of BP-related treatments between admission and EVT, use of sympathomimetics during EVT, weighted mean BP during EVT and rate of BP oscillations}

\begin{abstract}
Most subjects received no BP-related treatment between admission and EVT $(n=146)$, two subjects received sympathomimetics and 16 received BP-lowering treatment ( 15 received 5 to $75 \mathrm{mg}$ of urapidil intravenously, 1 received $50 \mathrm{mg}$ urapidil+ $0.075 \mathrm{mg}$ clonidine subcutaneously). The latter subset was characterized by numerically higher on-admission systolic blood pressure (SBP) and mean arterial pressure (MAP) and a greater average within-subject decline in SBP and MAP between admission and EVT (reference BP values appeared comparable across subsets) (Table A). Similar proportions of subjects received sympathomimetics during EVT (Table A).

We undertook the following analyses:

1. Identification of on-admission characteristics associated with the use of antihypertensives between admission and EVT

2. Identification of factors associated with the extent of blood pressure change between admission and start of EVT

3. Relationship between on-admission BP and BP at the start of EVT ("reference BP")

4. Identification of on-admission characteristics and factors occurring between admission and EVT associated with the use of sympathomimetics during EVT

5. Identification of factors associated with weighted mean BP during EVT and BP excursions to $>120 \%$ or $<80 \%$ of the reference BP during EVT
\end{abstract}

1. On-admission characteristics associated with the use of antihypertensives between admission and EVT

The use of antihypertensive treatment between admission and start of EVT was considered a binary dependent variable (the two patients who received sympathomimetics were considered as "not treated with antihypertensives"), and was analyzed in a logistic model. It included three default independent variables: on-admission SBP, on-admission MAP, and clinical stroke severity on admission (National Institutes of Health Stroke Scale [NIHSS] score). Demographics, medical history, comorbidity, type of affected vessel (dichotomized as middle cerebral artery segment 1 vs. other) and whether recombinant tissue plasminogen activator (rtPA) was used along EVT were considered as potential independent variables through a stepwise selection procedure with $P<0.200$ to enter/ stay in the model. Results are summarized in Table B. Higher on-admission SBP was associated with higher odds of being administered antihypertensives, while pre-stroke anticoagulant use and middle cerebral artery segment 1 occlusion were associated with lower odds. MAP and stroke severity at presentation did not appear associated with the odds of administration of antihypertensives between admission and EVT.

2. Factors associated with the extent of BP change between admission and start of EVT

The extent of intra-individual BP change between admission and start of EVT ( $\triangle$ reference - on-admission BP) was considered a dependent variable, separately for SBP and MAP. Two models were fitted-Model 1 did not include on-admission BP value, while Model 2 included this adjustment as well. Model 1 included two default independent variables-age and antihypertensive treatment; Model 2 included three default independent variables-age, use of antihypertensives and on-admission BP value. Other effects were selected through backward elimination $(P<0.200)$ from a full model including demographic and medical history data, use of rtPA, time-lag between admission BP and reference BP measurement, affected vessel and on-admission NIHSS score. Results are summarized in Table C. When not accounting for on-admission BP (Table C, Model 1), patients who received antihypertensive treatment between admission and start of EVT experienced a considerably greater reduction in BP (vs. those who did not): by around $26 \mathrm{~mm} \mathrm{Hg}$ greater reduction in SBP and by around $15 \mathrm{~mm}$ $\mathrm{Hg}$ greater reduction in MAP. There appeared also a tendency towards a greater BP reduction in older subjects. However, when on-admission BP was taken into consideration (Table $C_{1}$ Model 2), the difference between antihypertensive-treated and not-treated patients was several-fold reduced and appeared minor. The effect of age was also reduced. By $1 \mathrm{~mm} \mathrm{Hg}$ higher on-admission BP (both SBP and MAP) was associated with by close to $1 \mathrm{~mm} \mathrm{Hg}$ greater BP reduction.

\section{Relationship between on-admission BP and BP at the start of EVT ("reference BP")}

To evaluate the relationship between on-admission and reference BP (In-transformed dependent variable), a model was fitted to data with on-admission NIHSS score, use of antihypertensives between admission and EVT and on-admission BP as independent variables. On-admission values were mean-centered (to avoid collinearity) since the model explored potential linear and quadratic relationship, and also included interaction terms between on-admission BP and use of antihypertensives to assess potential dissimilarities between on-admission and reference BP relationship in patients treated and not treated with antihypertensives. Results are summarized in Table D. Figure B shows scatterplot of individual data and adjusted linear and quadratic regression lines overall and by subsets of patients in respect to antihypertensive treatment between admission and start of EVT (separately for SBP and MAP). Considering both SBP and MAP, there appeared no association between on-admission NIHSS score and reference BP, while differences between patients who received and who did not receive antihypertensives between admission and EVT were minor (Table D).

Regarding SBP, there was a linear association between higher on-admission and higher reference systolic $B P(P=0.024)$ and a stronger quadratic association $(P=0.004)$ (Table D). Adjusted linear regression lines are depicted in Figure BA. The interaction terms between on-admission BP and antihypertensive treatment were insignificant suggesting a similar relationship between on-admission and reference BP in patients not treated and treated with antihypertensives (Table D). In both subsets, there was a linear and a stronger quadratic association between on-admission and reference BP (Table D). Adjusted regression lines are depicted in Figure BB.

Regarding MAP, graphically (Figure BA), there appeared a linear and a quadratic association between on-admission BP and reference BP, but in this model neither appeared significant (Table D). Interaction terms between antihypertensive treatment and on-admission BP did not indicate substantial differences in on-admission-to-reference BP relationship in subsets of patients treated or not treated with antihypertensives between admission and EVT. Numerically, coefficients were similar (Table D), but a "near-significant" linear $(P=0.055)$ and a stronger quadratic $(P=0.019)$ relationship was observed only in the larger subset of non-treated patients (Table D). Adjusted regression lines for the two subsets of patients are depicted in Figure BB.

4. Factors between admission and EVT associated with the use of sympathomimetics during EVT

Logistic model was fitted to a binary dependent variable "sympathomimetic use during EVT" with reference BP (systolic, MAP), age, admission NIHSS, use of rtPA, use of antihypertensives between admission and EVT, type of affected vessel and use of antihypertensives type of affected vessel interaction. Results are summarized in Table $\mathrm{E}$. 
There appeared no association between the use of sympathomimetics during EVT and reference systolic BP or MAP, stroke severity, use of rtPA, use of antihypertesives before EVT and age. Subjects suffering a middle cerebral artery (MCA) 1 stroke were less likely to receive sympathomimetics during EVT -similarly in patients who received and who did not receive antihpyertensives before EVT.

5. Factors associated with weighted mean BP during EVT and BP excursions to $>120 \%$ or $<80 \%$ of the reference BP

General linear models were fitted to weighted mean systolic BP/MAP during EVT. Higher reference BP and higher rate of BP excursions to $>120 \%$ of the reference during EVT were independently associated with higher mean SBP/MAP (Table F). The coefficient for the interaction term indicated that the "effect" of excursions was higher at higher reference BP (understandably). Higher rate of BP excursions to $<80 \%$ was independently associated with lower mean SBP/ MAP during EVT (Table F). The interaction between the rate of excursions to $<80 \%$ and reference BP was highly insignificant and was removed. Men tended to have lower mean BP during EVT than women (Table F). No association was observed between age, on-admission NIHSS score, pre-EVT use of antihypertensives, use or rtPA, type of affected vessel, procedure duration, number of BP measurements taken and use of sympathomimetics during EVT and the mean BP during the procedure (Table F).

Poisson regression models were fitted to the rate of SBP/MAP excursions to $>120 \%$ and to $<80 \%$ of the reference BP. Higher reference SBP was independently associated with a lower risk of SBP excursions to $>120 \%$ and a higher risk of excursions to $<80 \%$ (Table G). The same pattern of associations was observed for reference MAP and MAP excursions during EVT (Table G). For both SBP and MAP, use of sympathomimetics during EVT tended towards association with a higher risk of excursions to $>120 \%$ and a lower risk of excursions to $<80 \%$ (Table G). Pre-EVT use of antihypertensives was associated with lower risk of SBP excursions to $>120 \%$ and tended to a higher risk of excursions to $<80 \%$ (Table G). For both SBP and MAP, rtPA use tended to association with a higher risk of excursions to $>120 \mathrm{~mm} \mathrm{Hg}$ and a lower risk of excursions to $<80 \%$ (Table $\mathrm{G}$ ).

The present analyses indicate: (a) on-admission BP appeared the main driver of a decision to administer antihypertensives before EVT, regardless of the stroke severity, likely in order to achieve recommended BP levels for the reperfusion procedure; (b) the predominant decline in BP between admission and start of EVT was only partly ascribable to administered antihypertensives since it occurred to a similar extent in patients not treated with antihypertensives, likely due to calming/induction of general anesthesia. It did not appear associated with the stroke severity; (c) reference BP appeared the main factor guiding the "tolerance" towards BP excursions during EVT (and, hence, overall weighted mean BP) independently of the means by which it was achieved (i.e., with or without pre-EVT antihypertensive use)-higher reference was strongly associated with a lower risk of excursions to higher values and a higher risk of excursions to lower values (vs. the reference). The rate of oscillations did not appear associated with clinical stroke severity. The use of sympathomimetics during EVT appeared associated with BP excursions just in a way opposite to reference BP. Overall data suggest that BP-related measures (antihypertensives, sympathomimetics, "tolerance" towards excursions) were guided predominantly by the intention to ascertain BP values within the recommended limits for reperfusion procedures, and not by stroke characteristics. 
Table A. Subject characteristics in respect to received blood pressure-related treatment between admission and the start of endovascular thrombectomy

\begin{tabular}{|c|c|c|c|}
\hline Characteristic & $\begin{array}{c}\text { No BP-related treatment between } \\
\text { admission and EVT }\end{array}$ & $\begin{array}{l}\text { BP-lowering treatment between } \\
\text { admission and EVT }\end{array}$ & $\begin{array}{c}\text { Sympathomimetics between ad- } \\
\text { mission and EVT }\end{array}$ \\
\hline Number & 146 & 16 & 2 \\
\hline Age (yr) & 74 (20 to 92$)$ & 76 (53 to 90$)$ & 44 to 79 \\
\hline Male sex & $66(45.2)$ & $7(43.8)$ & 0 \\
\hline Atrial fibrillation & $58(39.7)$ & $5(31.3)$ & 1 \\
\hline History of hypertension & $95(65.1)$ & $11(68.8)$ & 0 \\
\hline Previous stroke & $17(11.6)$ & $0(0)$ & 1 \\
\hline Peripheral artery disease & $11(7.5)$ & $0(0)$ & 0 \\
\hline Ischemic heart disease & $31(21.2)$ & $3(18.8)$ & 1 \\
\hline Carotid stenosis $\geq 50 \%$ & $15(10.3)$ & $2(12.5)$ & 0 \\
\hline Chronic heart failure & $25(17.5)$ & $1(6.3)$ & 0 \\
\hline Pre-admission anticoagulants & 27 (18.5) & $1(6.3)$ & 1 \\
\hline Middle cerebral artery segment 1 & $104(71.2)$ & $8(50.0)$ & 2 \\
\hline Middle cerebral artery segment 2 & $12(8.2)$ & $2(12.5)$ & 0 \\
\hline Tandem occlusion & $30(20.6)$ & $6(37.5)$ & 0 \\
\hline Admission SBP (mm Hg) & 150 (83 to 220$)$ & 178 (120 to 223$)$ & 100 to 120 \\
\hline Admission MAP (mm Hg) & 107 (56 to 167$)$ & 115 (97 to 174$)$ & 73 to 95 \\
\hline Admission NIHSS (score) & 18 (3 to 32 ) & 19 (9 to 32 ) & 16 to 22 \\
\hline Lag: admission-reference BP (min) & 24 (1 to 68$)$ & 26 (7 to 45$)$ & 25 to 52 \\
\hline Reference SBP (mm Hg) & 125 (73 to 203$)$ & 121 (95 to 174$)$ & 116 to 139 \\
\hline SPB $\triangle$ reference-admission $(\mathrm{mm} \mathrm{Hg})$ & $-22(-105$ to 66$)$ & $-42(-126$ to 0$)$ & -4 to 39 \\
\hline Reference MAP (mm Hg) & 89 (45 to 136$)$ & 84 (63 to 124$)$ & 83 to 100 \\
\hline MAP $\triangle$ reference-admission $(\mathrm{mm} \mathrm{Hg})$ & $-16(-101$ to 50$)$ & $-29(-83$ to 8$)$ & -12 to 27 \\
\hline Use of rtPA & $103(70.6)$ & $12(75.0)$ & 1 \\
\hline Sympathomimetics during EVT & $67(45.9)$ & $9(56.3)$ & 1 \\
\hline EVT weighted mean SBP ( $\mathrm{mm} \mathrm{Hg}$ ) & 128 (69 to 192$)$ & 125 (99 to 155$)$ & 109 to 127 \\
\hline EVT weighted mean MAP $(\mathrm{mm} \mathrm{Hg})$ & 92 (43 to 125$)$ & 89 (67 to 106$)$ & 82 to 96 \\
\hline \multicolumn{4}{|l|}{ Rates of BP excursions ( $\mathrm{n} / 10 \mathrm{~min}$ ) } \\
\hline SBP $>120 \%$ of reference & 1.17 (0 to 9.26$)$ & $0.72(0$ to 3.75$)$ & 0 to 0.35 \\
\hline MAP $>120 \%$ of reference & 1.18 (0 to 9.74$)$ & 1.15 (0 to 4.0$)$ & 0 to 2.62 \\
\hline SBP $<80 \%$ of reference & 0.95 (0 to 9.20$)$ & 0.83 (0 to 7.79$)$ & 0 to 7.03 \\
\hline MAP $<80 \%$ of reference & 0.75 (0 to 8.92$)$ & $0.90(0$ to 8.21$)$ & 0 to 6.2 \\
\hline
\end{tabular}

Values are presented as median (range), geometric mean (range) for rates of BP excursions, and count (percent). Individual data are shown for two subjects who received sympathomimetics between admission and EVT.

BP, blood pressure; EVT, endovascular thrombectomy; SBP, systolic blood pressure; MAP, mean arterial pressure; NIHSS, National Institutes of Health Stroke Scale; rtPA, recombinant tissue plasminogen activator. 
Table B. Summary of multivariate analysis of the outcome "antihypertensive treatment between admission and endovascular thrombectomy"

\begin{tabular}{llc}
\hline Variable & OR (95\% Cl) & $P$ \\
\hline Default independent variables & $1.47(1.01-2.22)$ & 0.045 \\
On-admission SBP (by $10 \mathrm{~mm} \mathrm{Hg})$ & $0.95(0.54-1.63)$ & 0.858 \\
On-admission mean arterial pressure (by $10 \mathrm{~mm} \mathrm{Hg})$ & $1.04(0.95-1.15)$ & 0.405 \\
On-admission NIHSS score (by 1 score point) & $0.15(0.01-0.97)$ & 0.045 \\
Selected independent variables & $0.35(0.11-1.10)$ & 0.072 \\
Pre-stroke anticoagulant use (vs. none) &
\end{tabular}

$\mathrm{OR}$, odds ratio; $\mathrm{Cl}$, confidence interval; $\mathrm{SBP}$, systolic blood pressure; NIHSS, National Institutes of Health Stroke Scale.

Table C. Summary of multivariate analysis of the outcome "change in BP between admission and start of endovascular thrombectomy"

\begin{tabular}{|c|c|c|c|c|}
\hline \multirow{2}{*}{ Variable } & \multicolumn{2}{|c|}{ Change in SBP } & \multicolumn{2}{|c|}{ Change in MAP } \\
\hline & $\Delta$ Change $(95 \% \mathrm{Cl})$ & $P$ & $\Delta$ Change $(95 \% \mathrm{Cl})$ & $P$ \\
\hline \multicolumn{5}{|l|}{ Model 1 (not accounting for admission BP) } \\
\hline Received antihypertensive treatment (vs. no) & $-25.6(-41.6$ to -9.6$)$ & 0.002 & $-14.5(-25.9$ to -3.0$)$ & 0.014 \\
\hline Age (by $10 \mathrm{yr}$ ) & $-2.6(-6.0$ to 0.7$)$ & 0.127 & $-2.3(-4.7$ to 0.1$)$ & 0.065 \\
\hline \multicolumn{5}{|l|}{ Model 2 (accounting for admission BP) } \\
\hline \multicolumn{5}{|l|}{ Default independent variables } \\
\hline Received antihypertensive treatment (vs. no) & $-6.1(-18.2$ to 5.9$)$ & 0.315 & $-2.7(-11.3$ to -5.8$)$ & 0.529 \\
\hline Age (by 10 yr) & $-1.3(-3.9$ to 1.2$)$ & 0.300 & $-0.4(-2.2$ to 1.4$)$ & 0.678 \\
\hline On-admission BP (by $1 \mathrm{~mm} \mathrm{Hg}$ ) & $-0.9(-1.0$ to -0.7$)$ & $<0.001$ & $-0.9(-1.0$ to -0.7$)$ & $<0.001$ \\
\hline \multicolumn{5}{|l|}{ Selected independent variables } \\
\hline Men (vs. women) & - & - & $-4.1(-9.2$ to 0.9$)$ & 0.105 \\
\hline Pre-existing hypertension (vs. no) & - & - & $-3.8(-9.0$ to 1.5$)$ & 0.161 \\
\hline
\end{tabular}

$\mathrm{BP}$, blood pressure; $\mathrm{SBP}$, systolic blood pressure; MAP, mean arterial pressure; $\mathrm{Cl}$, confidence interval.

Table D. Summary of multivariate analysis of "reference BP" (separately for systolic and mean arterial pressure)

\begin{tabular}{|c|c|c|c|c|}
\hline \multirow{2}{*}{ Variable } & \multicolumn{2}{|c|}{ Systolic blood pressure } & \multicolumn{2}{|l|}{ Mean arterial pressure } \\
\hline & $\beta(95 \% \mathrm{Cl})$ & $P$ & $\beta(95 \% \mathrm{Cl})$ & $P$ \\
\hline On-admission NIHSS score (by 5 points) & $0.013(-0.009$ to 0.036$)$ & 0.234 & $0.001(-0.024$ to 0.025$)$ & 0.920 \\
\hline Received antihypertensives (vs. no) & 0.024 (-0.096 to 0.144$)$ & 0.697 & -0.041 ( -0.149 to 0.068$)$ & 0.461 \\
\hline On-admission BP (by $10 \mathrm{~mm} \mathrm{Hg}$ ) & 0.026 (0.003 to 0.049$)$ & 0.024 & $0.021(-0.028$ to 0.070$)$ & 0.398 \\
\hline On-admission $\mathrm{BP}^{2}$ (by $100 \mathrm{~mm} \mathrm{Hg}$ ) & $-0.007(-0.012$ to -0.002$)$ & 0.004 & $-0.005(-0.014$ to 0.004$)$ & 0.280 \\
\hline On-admission BP*antihypertensives & $0.024(-0.021$ to 0.069$)$ & 0.287 & $0.008(-0.089$ to 0.104$)$ & 0.870 \\
\hline On-admission $\mathrm{BP}^{2} *$ antihypertensives & $-0.006(-0.016$ to 0.004$)$ & 0.208 & $0.002(-0.017$ to 0.021$)$ & 0.825 \\
\hline BP when no antihypertensive treatment & 0.014 (0.003 to 0.025$)$ & 0.013 & 0.017 (0.000 to 0.034$)$ & 0.055 \\
\hline BP when antihypertensive treatment & $0.038(-0.006$ to 0.082$)$ & 0.086 & $0.025(-0.070$ to 0.012$)$ & 0.607 \\
\hline $\mathrm{BP}^{2}$ when no antihypertensive treat. & $-0.004(-0.007$ to -0.001$)$ & 0.007 & $-0.006(-0.011$ to -0.001$)$ & 0.019 \\
\hline $\mathrm{BP}^{2}$ when antihypertensive treatment & $-0.011(-0.020$ to -0.001$)$ & 0.029 & $-0.004(-0.022$ to 0.014$)$ & 0.654 \\
\hline
\end{tabular}

Models are fitted to In-transformed reference $B P$ values.

$\mathrm{BP}$, blood pressure; $\mathrm{Cl}$, confidence interval; $\mathrm{BP}^{2}$, blood pressure by $100 \mathrm{~mm} \mathrm{Hg}$; NIHSS, National Institutes of Stroke Scale. 
Table E. Summary of multivariate analysis of the outcome "sympathomimetic use during EVT"

\begin{tabular}{llc}
\hline & OR (95\% Cl) & $P$ \\
\hline Systolic BP at start of EVT (by $10 \mathrm{~mm} \mathrm{Hg})$ & $1.12(0.83-1.53)$ & 0.449 \\
Mean arterial pressure at start of EVT (by $10 \mathrm{~mm} \mathrm{Hg})$ & $0.83(0.54-1.25)$ & 0.376 \\
Age (by $10 \mathrm{yr})$ & $1.00(0.78-1.28)$ & 0.999 \\
On-admission NIHSS (by 5 points) & $1.14(0.85-1.53)$ & 0.393 \\
Use of rtPA (vs. no) & $0.72(0.35-1.47)$ & 0.373 \\
Use of antihypertensive before EVT (vs. no) & $1.18(0.66-2.18)$ & 0.577 \\
Middle cerebral artery segment 1 (vs. other) & $0.56(0.30-0.97)$ & 0.041 \\
Antihypertensive use*affected vessel & $0.73(0.38-1.30)$ & 0.293 \\
Use of antihypertensives at MCA1 & $0.74(0.14-3.34)$ & - \\
Use of antihypertensives at "other vessel" & $2.59(0.59-20.1)$ \\
MCA1 vs. "other" at antihypertensives use & $0.17(0.01-1.41)$ & - \\
MCA1 vs. "other" at no antihypertensive use & $0.58(0.27-1.23)$ \\
\hline
\end{tabular}

$\mathrm{EVT}$, endovascular thrombectomy; $\mathrm{OR}$, odds ratio; $\mathrm{Cl}$, confidence interval; $\mathrm{BP}$, blood pressure; NIHSS, National Institutes of Health Stroke Scale; rtPA, recombinant tissue plasminogen activator; MCA, middle cerebral artery.

Table F. Summary of multivariate analyses of weighted mean SBP and MAP during EVT

\begin{tabular}{|c|c|c|c|c|}
\hline \multirow{2}{*}{ Variable } & \multicolumn{2}{|c|}{ Weighted mean SBP } & \multicolumn{2}{|c|}{ Weighted mean MAP } \\
\hline & $\beta(95 \% \mathrm{Cl})$ & $P$ & $\beta(95 \% \mathrm{Cl})$ & $P$ \\
\hline Reference BP $(\mathrm{mm} \mathrm{Hg})$ & 0.76 (0.66 to 0.86$)$ & $<0.001$ & 0.80 (0.71 to 0.89 ) & $<0.001$ \\
\hline Rate: $B P>120 \%$ reference $(\mathrm{n} / 10 \mathrm{~min})$ & 5.11 (3.99 to 6.24$)$ & $<0.001$ & 3.58 (2.87 to 4.29$)$ & $<0.001$ \\
\hline Reference $\mathrm{BP} *$ rate $>120 \%$ & 0.08 (0.02 to 0.15$)$ & 0.008 & 0.07 (0.03 to 0.10$)$ & $<0.001$ \\
\hline Rate: $\mathrm{BP}<80 \%$ reference $(\mathrm{n} / 10 \mathrm{~min})$ & $-4.96(-5.91$ to -4.02$)$ & $<0.001$ & $-3.48(-4.16$ to -2.80$)$ & $<0.001$ \\
\hline Age (by $10 \mathrm{yr}$ ) & $0.21(-1.01$ to 1.42$)$ & 0.738 & $-0.21(-1.01$ to 0.59$)$ & 0.605 \\
\hline Men (vs. women) & $-3.41(-6.63$ to -0.18$)$ & 0.039 & $-1.82(-3.97$ to -0.32$)$ & 0.095 \\
\hline On-admission NIHSS score & $0.02(-0.27$ to 0.31$)$ & 0.894 & $0.10(-0.09$ to 0.29$)$ & 0.293 \\
\hline MCA1 (vs. "other" vessel) & $-1.50(-5.05$ to 2.06$)$ & 0.407 & $-1.80(-4.13$ to 0.55$)$ & 0.133 \\
\hline rtPA use (vs. no) & $-1.87(-5.47$ to 1.74$)$ & 0.308 & $-0.98(-3.40$ to 1.44$)$ & 0.425 \\
\hline Pre-EVT antihypertensives (vs. no) & $1.57(-3.83$ to 6.98$)$ & 0.566 & $1.44(-2.11$ to 5.01$)$ & 0.424 \\
\hline Sympathomimetics during EVT (vs. no) & $0.31(-2.91$ to 3.53$)$ & 0.850 & $0.17(-1.98$ to 2.32$)$ & 0.877 \\
\hline EVT duration (min) & $-0.01(-0.05$ to 0.03$)$ & 0.541 & $-0.00(-0.03$ to 0.02$)$ & 0.787 \\
\hline BP measurements during EVT (n) & $-0.01(-0.05$ to 0.04$)$ & 0.813 & $-0.01(-0.04$ to 0.03$)$ & 0.734 \\
\hline
\end{tabular}

SBP, systolic blood pressure; MAP, mean arterial pressure; EVT, endovascular thrombectomy; $\mathrm{Cl}$, confidence interval; $\mathrm{BP}$, blood pressure; NIHSS, National Institutes of Health Stroke Scale; MCA, middle cerebral artery; rtPA, recombinant tissue plasminogen activator. 
Table G. Summary of multivariate analyses of the "rate (n/10 min) of BP excursions to $>120 \%$ of the reference BP during EVT" and "rate of BP excursions to $<80 \%$ of the reference BP during EVT"

\begin{tabular}{|c|c|c|c|c|}
\hline \multirow{2}{*}{ Variable } & \multicolumn{2}{|c|}{ Rate to $>120 \%$} & \multicolumn{2}{|c|}{ Rate to $<80 \%$} \\
\hline & $\mathrm{RR}(95 \% \mathrm{Cl})$ & $P$ & $\mathrm{RR}(95 \% \mathrm{Cl})$ & $P$ \\
\hline \multicolumn{5}{|l|}{ Model of SBP/SBP excursions } \\
\hline Reference BP (by $10 \mathrm{~mm} \mathrm{Hg}$ ) & $0.66(0.61-0.72)$ & $<0.001$ & $1.63(1.52-1.76)$ & $<0.001$ \\
\hline Sympathomimetics during EVT (vs. no) & $1.21(0.89-1.62)$ & 0.220 & $0.67(0.45-0.98)$ & 0.040 \\
\hline Pre-EVT use of antihypertensives (vs. no) & $0.57(0.31-0.98)$ & 0.040 & $1.54(0.82-2.76)$ & 0.175 \\
\hline rtPA used (vs. no) & $1.46(1.04-2.08)$ & 0.029 & $0.81(0.52-1.26)$ & 0.338 \\
\hline Age (by 10 yr) & $1.10(0.98-1.24)$ & 0.095 & $0.86(0.75-0.99)$ & 0.036 \\
\hline Men (vs. women) & $1.09(0.81-1.46)$ & 0.576 & $1.21(0.81-1.81)$ & 0.340 \\
\hline On-admission NIHSS (by 10 points) & $0.99(0.76-1.29)$ & 0.965 & $0.77(0.54-1.10)$ & 0.150 \\
\hline MCA1 (vs. "other" vessel) & $0.73(0.53-1.03)$ & 0.072 & $0.76(0.51-1.12)$ & 0.163 \\
\hline EVT duration (by $10 \mathrm{~min}$ ) & $0.95(0.81-1.02)$ & 0.267 & $0.96(0.74-1.03)$ & 0.433 \\
\hline BP measurements during EVT (by 10) & $1.05(0.97-1.25)$ & 0.270 & $1.10(0.93-1.43)$ & 0.232 \\
\hline \multicolumn{5}{|l|}{ Model for MAP/MAP excursions } \\
\hline Reference BP (by $10 \mathrm{~mm} \mathrm{Hg}$ ) & $0.60(0.54-0.67)$ & $<0.001$ & $1.97(1.75-2.23)$ & $<0.001$ \\
\hline Sympathomimetics during EVT (vs. no) & $1.36(1.03-1.79)$ & 0.030 & $0.85(0.56-1.28)$ & 0.444 \\
\hline Pre-EVT use of antihypertensives (vs. no) & $0.70(0.40-1.14)$ & 0.156 & $0.97(0.48-1.83)$ & 0.930 \\
\hline rtPA used (vs. no) & $1.38(1.02-1.90)$ & 0.035 & $0.61(0.38-0.98)$ & 0.042 \\
\hline Age (by 10 yr) & $1.07(0.96-1.20)$ & 0.199 & $0.93(0.81-1.09)$ & 0.359 \\
\hline Men (vs. women) & $1.10(0.84-1.44)$ & 0.501 & $1.36(0.87-2.14)$ & 0.181 \\
\hline On-admission NIHSS (by 10 points) & $0.92(0.72-1.18)$ & 0.514 & $1.13(0.76-1.68)$ & 0.551 \\
\hline MCA1 (vs. "other" vessel) & $1.00(0.75-1.37)$ & 0.975 & $0.66(0.43-1.01)$ & 0.058 \\
\hline EVT duration (by $10 \mathrm{~min}$ ) & $0.95(0.84-1.02)$ & 0.232 & $0.95(0.73-1.01)$ & 0.130 \\
\hline BP measurements during EVT (by 10) & $1.05(0.97-1.20)$ & 0.266 & $1.09(0.93-1.41)$ & 0.211 \\
\hline
\end{tabular}

Four separate models were fitted: one for each rate, separately for SBP and MAP.

$\mathrm{BP}$, blood pressure; EVT, endovascular thrombectomy; RR, relative risk; $\mathrm{Cl}$, confidence interval; SBP, systolic blood pressure; rtPA, recombinant tissue plasminogen activator; NIHSS, National Institutes of Health Stroke Scale; MCA, middle cerebral artery; MAP, mean arterial pressure. 
All patients $(n=164)$
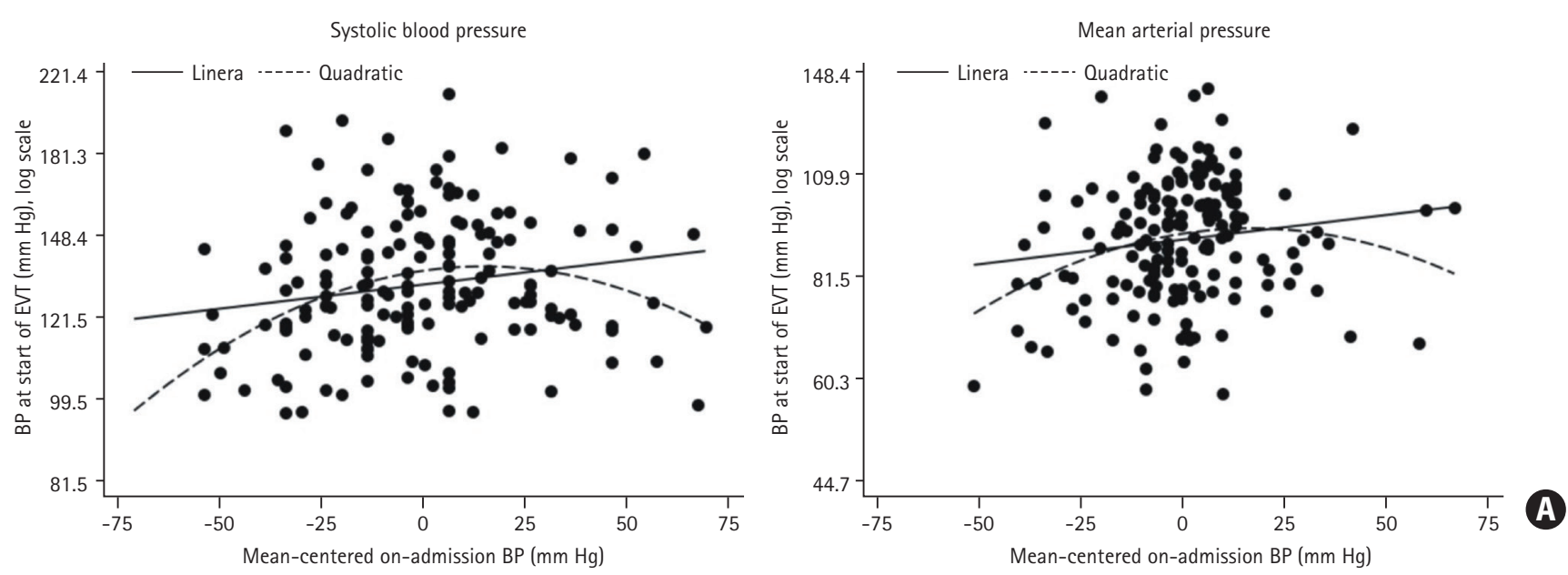

By antihypertensive treatment
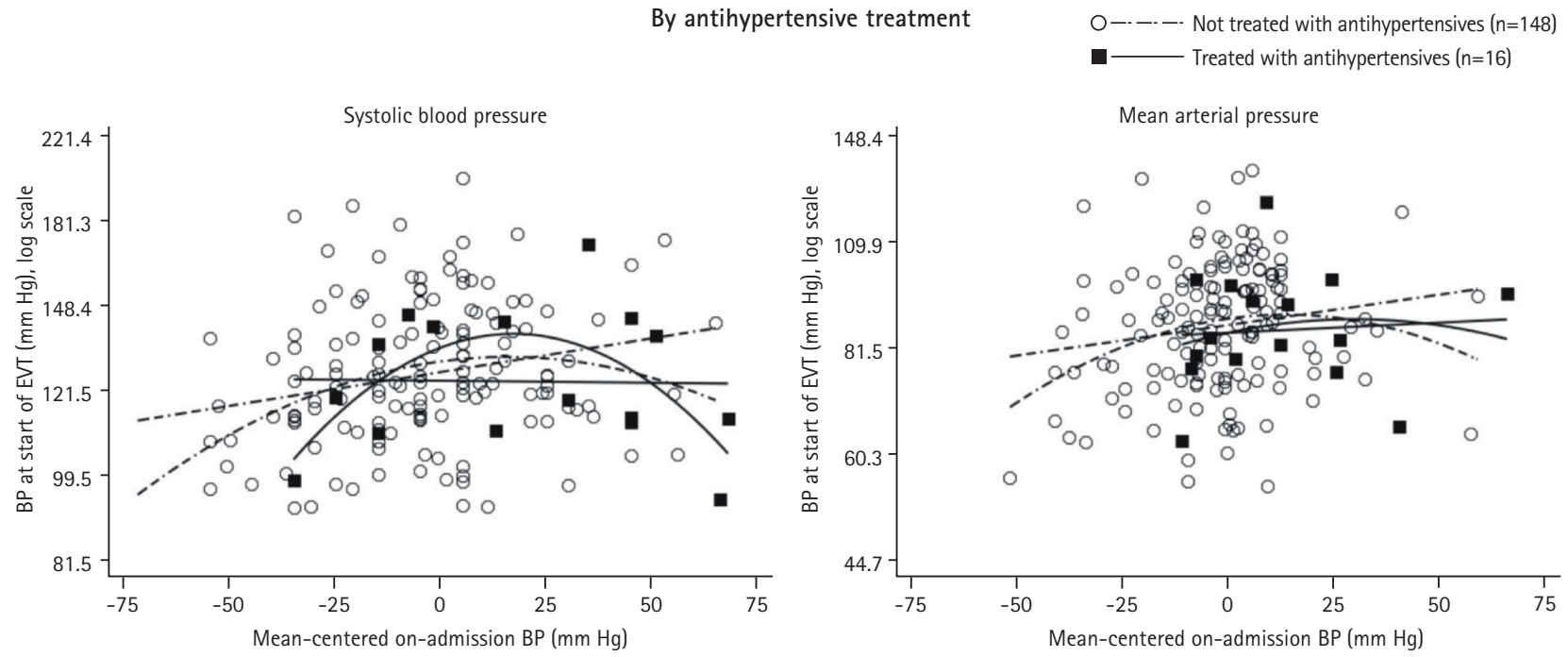

Figure B. Relationship between on-admission blood pressure (BP) and BP at the start of endovascular thrombectomy (EVT) (reference BP: mean of 3-5 values at anesthesia induction). (A) Overall (all patients). Closed circles are observed individual data, lines are adjusted regression lines. (B) By subset of patients in respect to administered antihypertensive treatment between admission and EVT. Symbols are observed individual data, lines are adjusted regression lines (both linear and quadratic). Since quadratic relationship was indicated by the initial analysis, mean-centered on-admission values were used (to avoid collinearity between linear and quadratic terms) in the main analysis. BP values at the beginning of EVT were In-transformed; hence, log scale is used at the y-axis. The model is depicted in Table $D$. 


\section{Supplementary Analysis of Post-Procedure Hemorrhages}

Analysis of the relationship between blood pressure (BP)/BP excursions during endovascular thrombectomy (EVT) to values $>120 \%$ or $<80 \%$ of the reference value and finding of visible hemorrhages on the post-procedure computed tomography scans (Supplementary Table 4) demonstrated a consistent lack of association between in-procedure BP excursions and the outcome (across a range of models). However, it disclosed an apparently counterintuitive finding: higher reference systolic blood pressure (SBP)/mean arterial pressure (MAP) consistently tended towards or was associated with higher odds of hemorrhages, whereas higher in-procedure mean BP was consistently associated lower odds of hemorrhages (Supplementary Table 4). Two observations indicated that the relationship between reference $\mathrm{BP}$, in-procedure mean $\mathrm{BP}$ and hemorrhages could be a complex one:

1. Strength of association between reference BP and hemorrhages and strength of association between in-procedure mean BP and hemorrhages appeared almost identical (but in an opposite direction). In the "reduced" model in Supplementary Table 4, odds ratio (OR) for the reference SBP was 1.48 and its inverse value $(0.67)$ is almost identical to the $O R$ for the procedure mean $S B P(O R=0.65)$. The same applies for reference $M A P(O R=1.86$; inverse value $=0.53)$ and in-procedure mean MAP $(\mathrm{OR}=0.52)$.

2. Supplemental analysis of BP (Table F) demonstrated that in-procedure mean BP was greatly determined by the reference BP.

We undertook the following analyses:

(a) We re-fitted the "reduced" model in Supplementary Table 4 with (i) inclusion of an interaction term between reference BP and in-procedure mean BP; (ii) with exclusion of the in-procedure mean $\mathrm{BP}$, while reference $\mathrm{BP}$ was retained; (iii) with exclusion of reference BP, while in-procedure mean BP was retained.

(b) We performed mediation analysis in which reference BP was considered a predictor, in-procedure mean was considered a mediator, presence of post-procedure hemorrhages was an outcome, while other effects from the "reduced" model in Supplementary Table 4 were covariates. All associations in the model, direct (predictor-mediator; mediator-outcome; predictor-outcome) and indirect (predictor-outcome, via mediator) were adjusted for all other effects; hence, all were independent.

\section{Re-fitted "reduced" model from Supplementary Table 4}

Table H summarizes results of re-fitting the "reduced" model from Supplementary Table 4. Only data for the reference BP and in-procedure mean BP are shown. All other effects (excursion rates, Thrombolysis in Cerebral Infarction [TICl] grade, stroke etiology, history of coronary artery disease) were consistently virtually identical as in Supplementary Table 4.

The interaction term between reference SBP/MAP and in-procedure mean SBP/MAP (mean-centered) was highly insignificant; however, in this model estimated effects of the reference BP on the odds of post-procedure hemorrhages were considerably changed as they became highly imprecise and statistically insignificant, whereas the effect of in-procedure mean BP remained closely similar as in the starting "reduced" model.

When in-procedure mean BP was removed from the model, reference BP was no longer associated with the odds of post-procedure hemorrhages. When reference BP was removed from the model, in-procedure mean BP was no longer associated with the odds of post-procedure hemorrhages.

\section{Mediation analysis}

Results are summarized in Table I. The results were consistent in the model for SBP and the model for MAP. Higher reference BP (predictor) was directly associated with higher in-procedure mean BP (mediator). This is in line with the results of the Supplementary Analysis of Blood Pressure (Table F). Higher in-procedure mean BP was directly associated with lower odds of post-procedure hemorrhages (with the adjustment for reference BP and other effects). This is line with the results of the "reduced" model in Supplementary Table 4. Higher reference BP (predictor) tended towards direct association with higher odds of postprocedure hemorrhages (outcome), but this association did not attain statistical significance. This is in line with the results of the "reduced" model in Supplementary Table 4. Namely, when a predictor and a mediator are simultaneously included in a "common" regression model (as is the "reduced" model in Supplementary Table 4), their individual direct associations with the outcome are quantified. Mediation analysis, using a set of consecutive regressions, partials-out direct and indirect associations (through a mediator) between the predictor and the outcome. As depicted in Table I, in both models (SBP/MAP), higher reference BP tended to be associated with lower odds of post-procedure hemorrhages, indirectly via its association with higher in-procedure mean BP, thus illustrating a phenomenon of "inconsistent mediation" (the direct and mediated effects are in an opposite direction). Consequently, in both models (SBP, MAP), the total effect (combined direct and indirect) of the reference BP (predictor) on the outcome was close to zero (direct and indirect effects mutually cancelledout). This is in line with the results of the re-fitted "reduced" model that did not include in-procedure mean BP (Table H): in a "common" regression model that does not include the mediator, effect of a predictor on the outcome corresponds to a total effect from the mediation analysis (i.e., the direct effect is not partialed-out).

Overall, the present analysis suggests that the observed opposite associations of the reference BP and of in-procedure mean BP with the probability of postprocedure hemorrhages in the "reduced" model in Supplementary Table 4, although apparently counterintuitive, can be explained by their mutual relationship. In terms of their practical meaning, the results of the "reduced" model in Supplementary Table 4 require cautious interpretation in which several facts need to be considered. Firstly, regarding the temporal sequence of events, reference BP precedes the in-procedure BP. Next, the two BP indices are driven by different factors. As shown in the Supplementary Analysis of Blood Pressure, reference BP results from (is defined by) on-admission BP and, in part, from measures undertaken in order to drive it into the limits recommended for the reperfusion procedure (Table D, Figure A). In-procedure mean BP, on the other hand, is largely determined by the reference BP in several ways: (a) higher reference BP is associated with higher in-procedure mean BP (Supplementary Analysis of Blood Pressure, Table F). This appears reasonable within the context of EVT: the procedure starts only after BP (reference BP) has been driven within the recommended boundaries, and BP is then maintained around this (preferred) value. Hence, higher the reference (within the recommendations)-higher the procedure mean $\mathrm{BP} ;(\mathrm{b})$ in-procedure mean $\mathrm{BP}$ is also largely determined by the rate of BP excursions-higher the rate of excursions to $>120 \%$ of the reference, higher the in-procedure mean; higher the rate of excursions to $<80 \%$, lower the in-procedure mean (Supplementary Analysis of Blood Pressure, Table F). Reference BP influences the in-procedure mean BP also by "driving" the rate of BP excursions: higher the reference $\mathrm{BP}$, lower the risk of $\mathrm{BP}$ excursions to $>120 \%$ of the reference and higher the risk of excursions to $<80 \%$ of the reference (Supplementary Analysis of Blood Pressure, Table G). Therefore, in the context of $\mathrm{EVT}$, with defined recommended pre-EVT BP values, reference BP is a milestone that defines the subsequent (during EVT) BP management, i.e., tolerance towards the oscillations, measures to reduce/control them. In this respect, the relationship between reference BP and post-procedure hemorrhages should preferably (as this is in line with the sequence of events) be viewed "through" the in-procedure mean BP. Hence, the main observation arising from the "reduced" model in Supplementary Table 4 is the association between higher in-procedure BP and lower odds of hemorrhages. Whatever effect reference BP "in itself" 
might have on the risk of post-procedure hemorrhages, this is cancelled-out by the subsequent in procedure BP: this is supported by the lack of a total effect of the reference BP in the mediation analysis due to opposing direct and mediated effects, and a lack of the effect of the reference BP in a "common" regression model when in-procedure mean is not accounted for (i.e., when the direct effect is not partialed-out from the total effect, i.e., when it is not separated from the indirect effect). This reasoning might be objected in the light of the fact that under similar conditions (re-fitted "reduced" model without an account for reference BP), in-procedure mean BP was also not associated with the odds of hemorrhages (Table H). In this respect, one should have in mind the specific temporal (reference BP precedes the in-procedure BP) and causal (reference BP determines in-procedure mean BP, and not vice versa) relationship between the reference and in-procedure BP. In this re-fitted model, one actually observes a "total" effect of in-procedure mean BP, i.e., this is a situation in which its specific direct effect on the risk of hemorrhages is not partialed-out from the total effect that it carries. Since it is cardinally determined by the reference BP, this total effect of the in-procedure mean BP actually largely represents the total effect of the reference BP (which is close to zero). It follows that in "common" regression models that exclude reference BP, one cannot actually identify the effect of in-procedure mean BP on the outcome (due to the strong causal relationship between the two).

Table H. Summary of the re-fitted versions of the "reduced" logistic model from Supplementary Table 4 analyzing association between reference BP and inprocedure weighted mean BP and occurrence of post-procedure hemorrhages

\begin{tabular}{|c|c|c|c|c|}
\hline \multirow{2}{*}{ Variable } & \multicolumn{2}{|c|}{ Systolic blood pressure } & \multicolumn{2}{|c|}{ Mean arterial pressure } \\
\hline & OR $(95 \% \mathrm{Cl})$ & $P$ & OR (95\% Cl) & $P$ \\
\hline Reference BP (by 10 mm Hg) & $1.48(0.99-2.28)$ & 0.056 & $1.86(1.00-3.47)$ & 0.051 \\
\hline In-procedure mean BP (by $10 \mathrm{~mm} \mathrm{Hg}$ ) & $0.65(0.40-0.96)$ & 0.032 & $0.52(0.26-0.95)$ & 0.034 \\
\hline \multicolumn{5}{|l|}{${ }^{+}$Reference $\mathrm{BP} *$ in-procedure mean $\mathrm{BP}$ interaction } \\
\hline Reference $\mathrm{BP} *$ in-procedure mean $\mathrm{BP}$ interaction & $0.99(0.98-1.00)$ & 0.206 & $1.00(0.98-1.01)$ & 0.669 \\
\hline Reference BP (by 10 mm Hg) & $3.94(0.81-19.0)$ & 0.088 & $2.66(0.45-15.6)$ & 0.278 \\
\hline In-procedure mean BP (by 10 mm Hg) & $0.64(0.42-0.99)$ & 0.046 & $0.51(0.27-0.96)$ & 0.039 \\
\hline \multicolumn{5}{|l|}{ In-procedure mean BP excluded } \\
\hline Reference BP (by $10 \mathrm{~mm} \mathrm{Hg}$ ) & $1.05(0.83-1.32)$ & 0.686 & $1.06(0.77-1.46)$ & 0.720 \\
\hline \multicolumn{5}{|l|}{ Reference BP excluded } \\
\hline In-procedure mean BP (by 10 mm Hg) & $0.89(0.71-1.14)$ & 0.379 & $0.88(0.65-1.21)$ & 0.441 \\
\hline
\end{tabular}

It is extension of model from Supplementary Table 4. In-procedure mean BP (by $10 \mathrm{~mm} \mathrm{Hg}$ ) is added to models from Supplementary Table 4, that's why there is a plus sign.

$\mathrm{BP}$, blood pressure; $\mathrm{OR}$, odds ratio; $\mathrm{Cl}$, confidence interval.

Table l. Summary of the mediation analysis: effects are shown as regression coefficients

\begin{tabular}{lr}
\hline Effects & $\beta(95 \% \mathrm{Cl}) ; P$ \\
\hline Model for SBP & 0.82 (0.73 to 0.91$) ;<0.001$ \\
Predictor (reference BP) $\rightarrow$ mediator (in-procedure mean BP) & $-0.0059(-0.0116$ to -0.0002$) ; 0.043$ \\
Mediator $\rightarrow$ outcome (odds of post-procedure hemorrhages) & $0.0056(-0.0001$ to 0.0113$) ; 0.056$ \\
Direct effect predictor $\rightarrow$ outcome & $-0.0048(-0.0094$ to 0.0015$) ; 0.078$ \\
Indirect effect predictor $\rightarrow$ outcome via mediator & $0.0007(-0.0026$ to 0.0040$) ; 0.661$ \\
Total effect (direct+indirect) predictor $\rightarrow$ outcome & \\
Model for MAP & $0.86(0.78-0.94) ;<0.001$ \\
Predictor (reference BP) $\rightarrow$ mediator (in-procedure mean BP) & $-0.0084(-0.0168$ to -0.0000$) ; 0.050$ \\
Mediator $\rightarrow$ outcome (odds of post-procedure hemorrhages) & $0.0082(-0.0005$ to 0.0169$) ; 0.060$ \\
Direct effect predictor $\rightarrow$ outcome & $-0.0073(-0.0150$ to 0.0020$) ; 0.099$ \\
Indirect effect predictor $\rightarrow$ outcome via mediator & $0.0010(-0.0035$ to 0.0055$) ; 0.678$ \\
Total effect (direct+indirect) predictor $\rightarrow$ outcome &
\end{tabular}

$\mathrm{Cl}$, confidence interval; SBP, systolic blood pressure; BP, blood pressure; MAP, mean arterial pressure. 